\title{
Implicit Large Eddy Simulation of Ship Airwakes
}

\author{
B. Thornber, M. Starr and D. Drikakis \\ Fluid Mechanics \& Computational Science Group, \\ Department of Aerospace Sciences, Cranfield University, \\ Cranfield, Bedfordshire, MK43 0AL, UK
}

\begin{abstract}
Implicit Large Eddy Simulations (ILES) of two different Royal Navy ships have been conducted as part of the UK Ship/Air Interface Framework project using a recently developed very high order accuracy numerical method. Time-accurate CFD data for fourteen flow angles was produced to incorporate into flight simulators for definition of safe helicopter operating limits (SHOLs). This paper discusses the flow phenomenology for the different wind directions and reports on the validation of the ILES results for mean and fluctuating velocity components and spectra against experimental data.
\end{abstract}

\section{Nomenclature}

$\begin{array}{ll}F & \text { Frequency } \mathrm{s}^{-1} \\ M & \text { Mach number } \\ P & \text { Power spectrum } \mathrm{m}^{2} \mathrm{~s}^{-3} \\ Q & \text { Vortex visualisation parameter } \mathrm{s}^{2} \\ R e & \text { Reynolds number } \\ u, v, w & \text { Wind components relative to WOD direction, } \mathrm{ms}^{-1} \\ u_{\text {cart }}, v_{\text {cart }}, w_{\text {cart }} & \text { Cartesian velocity components, } \mathrm{ms}^{-1} \\ u^{\prime}, v^{\prime}, w^{\prime} & \text { fluctuating velocity components, } \mathrm{ms}^{-1} \\ x, y, z & \text { Airwake coordinates, m } \\ \theta_{w} & \text { Wind direction, degrees } \\ \Delta x & \text { Mesh spacing, } \mathrm{m}\end{array}$




\section{Introduction}

This paper is concerned with the prediction of the time-varying airwake which is shed behind any ship which is moving relative to the wind. The behaviour of this airwake is of key importance to helicopter pilots, who must land safely on the flight deck in conditions which are potentially lethal. During the approach to the ship the pilots typically have to deal with adverse weather conditions, rolling and pitching of a very small landing deck and the presence of turbulence generated by the ships superstructure.

To enable pilots to decide if it is possible to land on a ship in a given weather condition, it is common practise to define 'Ship/Helicopter Operating Limits' (SHOLs) for a given ship-helicopter combination. The operating limits are given as a function of the wind over deck (WOD) velocity. Determining the SHOLs is extremely costly, requiring availability of the ship and test pilot time. The UK Ship/Air Interface Framework (SAIF) aims to enable experienced test pilots to predict the SHOLS for a given naval vessel years before the real test flights are undertaken and to give improvements to the quality of the operating limits, using advanced flight simulators. ${ }^{5}$

An important component in the SAIF project is the provision of a time-accurate airwake into the simulation framework. The aim was to verify if benefits can be gained by replacing the existing time-averaged data produced using Reynolds-Averaged Navier Stokes (RANS) computations. Although existing simulations included a fluctuating velocity added to the time averaged data, this fluctuating velocity could not be expected to have the correct spectral content as compared to a real airwake. In reality, the airwake consists of complex, interacting coherent structures. These coherent structures are, of course, dependent on the actual physical structure of a given ship.

A wide variety of numerical methodologies have been previously employed to simulate the high Reynolds number airwakes (typically $R e \approx 200 \times 10^{6}$ ). The majority of early and some recent simulations employ second order upwind methods with RANS modelling, where the mean flow field is captured and the unsteady data modelled. Tai ${ }^{44}$ produced RANS simulations with 1.3 million points, noting that the flow changes considerably as the wind angle changes. Reddy et al. ${ }^{34}$ used up to 1.5 millions grid points to simulate the Simple Frigate Shape (SFS). The results show strong grid dependence and even at the finest resolution the position vortex pair aft of the deck is incorrect. Bogstad et al. ${ }^{1}$ generated data for six different Royal Navy ships using a Finite Element method, showing good agreement with experimental data for the mean wind velocity for one wind direction. Polsky ${ }^{29}$ employed grid sizes up to 4 million points, demonstrating a significant improvement in predicting mean flow values when moving from RANS to unsteady Implicit Large Eddy Simulation (ILES).Sezer-Uzol et al. ${ }^{38}$ also noted the improvement in airwake quality gained 
in switching from steady to unsteady numerical methods. Syms ${ }^{43}$ used up to 0.9 million points demonstrating that RANS methods capture the correct flow features, however the gradients in the flow properties were too sharp. Most recent RANS results were presented by Roper et al. ${ }^{35}$ who computed flow around the SFS 1 \& 2 demonstrating reasonable agreement in mean flow quantities.

Building upon this experience in steady-state simulations, a consensus has been reached that for realistic representation of airwake flows a time accurate model must be employed. ${ }^{55}$ This permits two choices, Direct Numerical Simulation (DNS), or Large Eddy Simulation (LES). As the Reynolds number is on the order of $2 \times 10^{8}$ then DNS is not possible with the current computational power, thus all simulations to date have employed LES. As most previous papers (and the current contribution) do not employ an explicit subgrid model, they fall in to the class of Implicit Large-Eddy Simulation (ILES).

Applications of ILES to ship airwakes are numerous. ILES simulations by Guillot and Walker ${ }^{19}$ demonstrated good comparison of both mean flow quantities and spectral content using a second order method on 10 million grid points. Sharma and Long ${ }^{39}$ ran very coarse simulations with 0.2 million grid points achieved a good match to experimental trends. Polsky $^{29,30}$ has conducted numerous ILES simulation with up to 9 million points using a second order upwind method, showing a considerable improvement over RANS methods and demonstrating that the mean flow properties are reasonably invariant when scaled with respect to WOD. Polsky additionally highlighted the importance of using an atmospheric boundary layer profile in gaining more accurate results for side winds that are commonly referred to as beam winds. Czerwiec and Polsky ${ }^{6}$ investigated the same numerical method focusing on the bow of the ship, examining flow over the deck edge, gaining agreement with experimental data, noting that discrepancies could be due to scale effects (Reynolds number) inherent in wind tunnel experiments.

Results by Shipman et al. ${ }^{40}$ investigated the effects of geometrical representation of the ships on the quality of the airwake prediction, concluding that reasonable simplifications can be made to the ships superstructure without greatly affecting the majority of the airwake. Additionally, they note that including boundary layer resolving grid components only marginally improves the simulation quality. Preliminary unsteady Detached Eddy Simulation (DES) results have been presented by Forrest et al. ${ }^{12}$ illustrating the improvement in going from RANS to DES. Most recently Zhang et al. ${ }^{56}$ presented an ILES analysis of unsteady flow over the SFS2 giving a good agreement with experimental results in terms of mean velocities and spectra.

The current work differs notably from previous studies in that it employs a very high order accurate structured multi-block method instead of an unstructured approach. Although it is more complex, a grid can typically be generated in approximately two weeks given a 
clean CAD model and the structured grid approach allows the easy application of very high order accurate methods. Two different Royal Navy ships are examined, the Type 23 Frigate and the Wave Class Auxiliary Oiler. To validate the simulations, there is comparison with experimental and full-scale data provided by Qinetiq. ${ }^{33}$

The vast majority of the previous works use compressible upwind numerical methods to simulate the flow field, usually rescaling the flow velocity up to Mach 0.3. Most simulations run at Mach 0.3 because a) it is more computationally efficient (i.e. a smaller number of time steps per simulation) and b) upwind methods are excessively dissipative at low Mach numbers. ${ }^{51}$ With regard to the second reason, it has been shown that the dissipation of turbulent kinetic energy in an upwind scheme increases as the inverse of the Mach number. ${ }^{46}$ Hence if the simulation was run at the true Mach number then the upwind method would dissipate the majority of the turbulent structures. However, even when rescaling the flow Mach number to 0.3 , the instabilities which seed turbulent fluctuations occur at a much lower Mach number, meaning that they will still be excessively damped. This prevents the natural turbulent breakdown of large structures and the growth of very small structures, producing very dissipative results. ${ }^{47}$

Simulations reported here employ a very recently developed fifth order accurate method which has been modified to provide significantly improved behaviour in low Mach number turbulent flows. ${ }^{46}$ This involves a modification to the reconstruction method such that the dissipation rate is constant with Mach number, improving the effective resolution of the numerical scheme. Validation cases have been presented in Thornber et al. ${ }^{45,48}$ and Drikakis et al. ${ }^{10}$ This approach alleviates the overly dissipative behaviour of standard upwind methods, however it is still computationally more efficient (in terms of the number of time steps taken) to run the simulation at Mach 0.3 rather than the true Mach number.

The layout of this paper is as follows. Section II discusses the computational methods employed, including the grid generation, numerical methods and boundary conditions. Next, Section III and IV present the comparison with experiment for both ships for mean and fluctuating velocity components and discuss qualitatively the influence of WOD angle on the resultant flow field. Finally, conclusions are drawn in Section V.

\section{Computational Methods}

\section{II.A. Grid Generation}

Each ship was discretised using a curvilinear (body-fitted) multiblock method. By constructing the grids as a series of blocks (separate grids), this allows accurate representation of very complex geometries. The key advantage over unstructured grids is the use of high resolution and high order numerical methods which can capture complex flow features more accurately. 
The commercial software 'Gridgen' was employed to generate the grids. Note that in generating the grids no attempt was made to model the boundary layer attached to the ship. This simplification of the meshing requirements makes the prediction of flow separation from gradually curved surfaces less accurate, however previous studies by Shipman et al. ${ }^{40}$ investigated in depth the effects of resolving the boundary layer and concluded it has minimal influence on the quality of the wake prediction. Resolving the boundary layer would either make the computations prohibitively expensive, or remove grid points from more important regions of the flow such as the free shear layers and recirculation zones produced from sharp edges on the deck or hangar.

The computational domain was chosen to be in the shape of a cylinder of $400 \mathrm{~m}$ radius and $200 \mathrm{~m}$ altitude centred on the middle of the hangar door. This is shown schematically in Fig. 1. It allows for dynamic specification of the boundary conditions so that the wind direction can be changed easily from simulation to simulation.

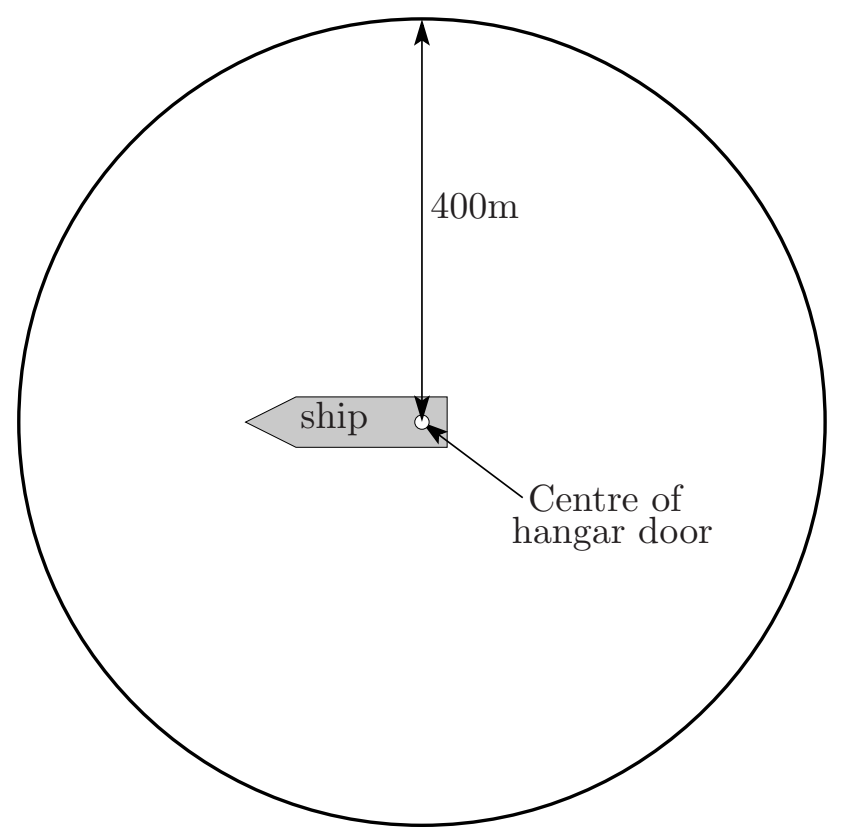

(a) Plan view

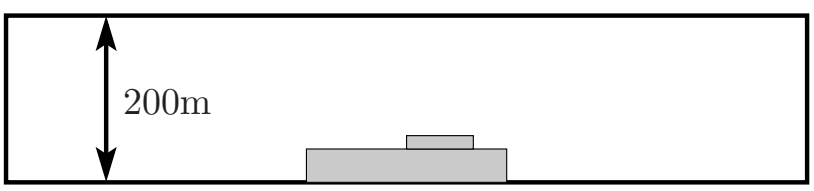

(b) Side View

Figure 1. Computational Domain 


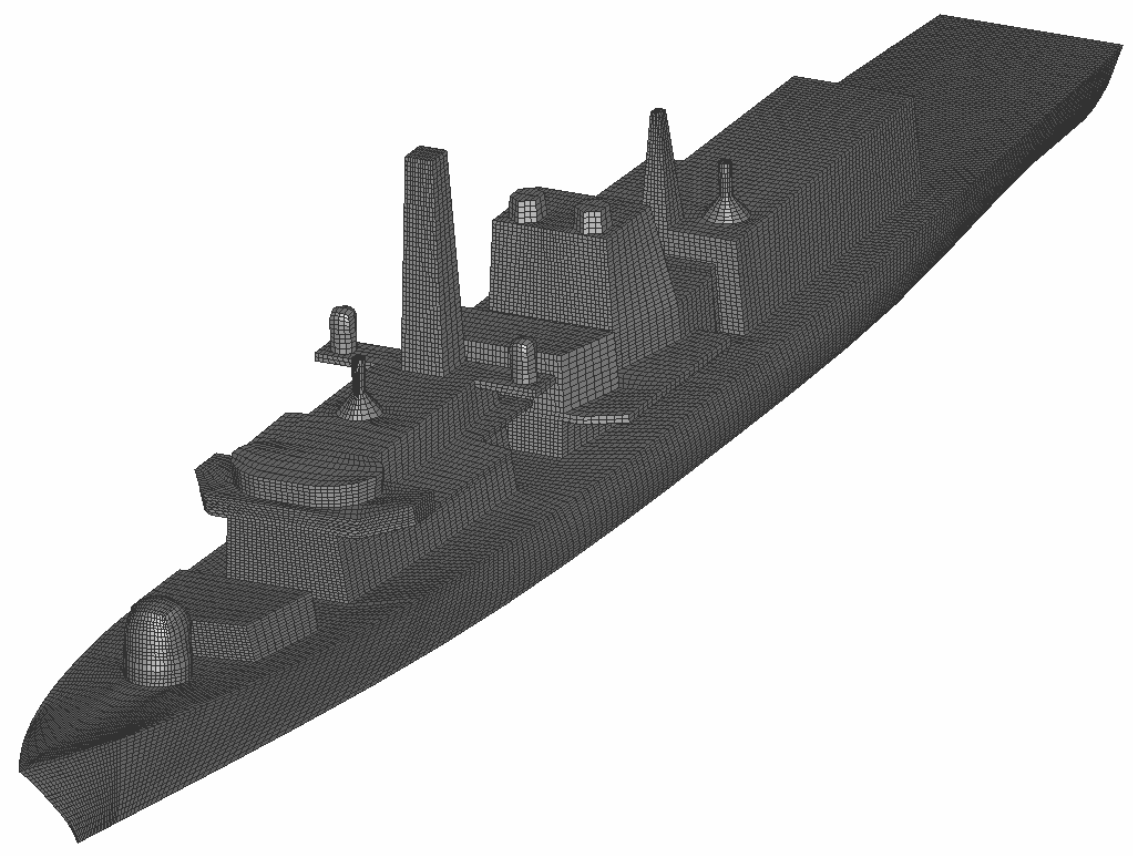

(a) Type 23

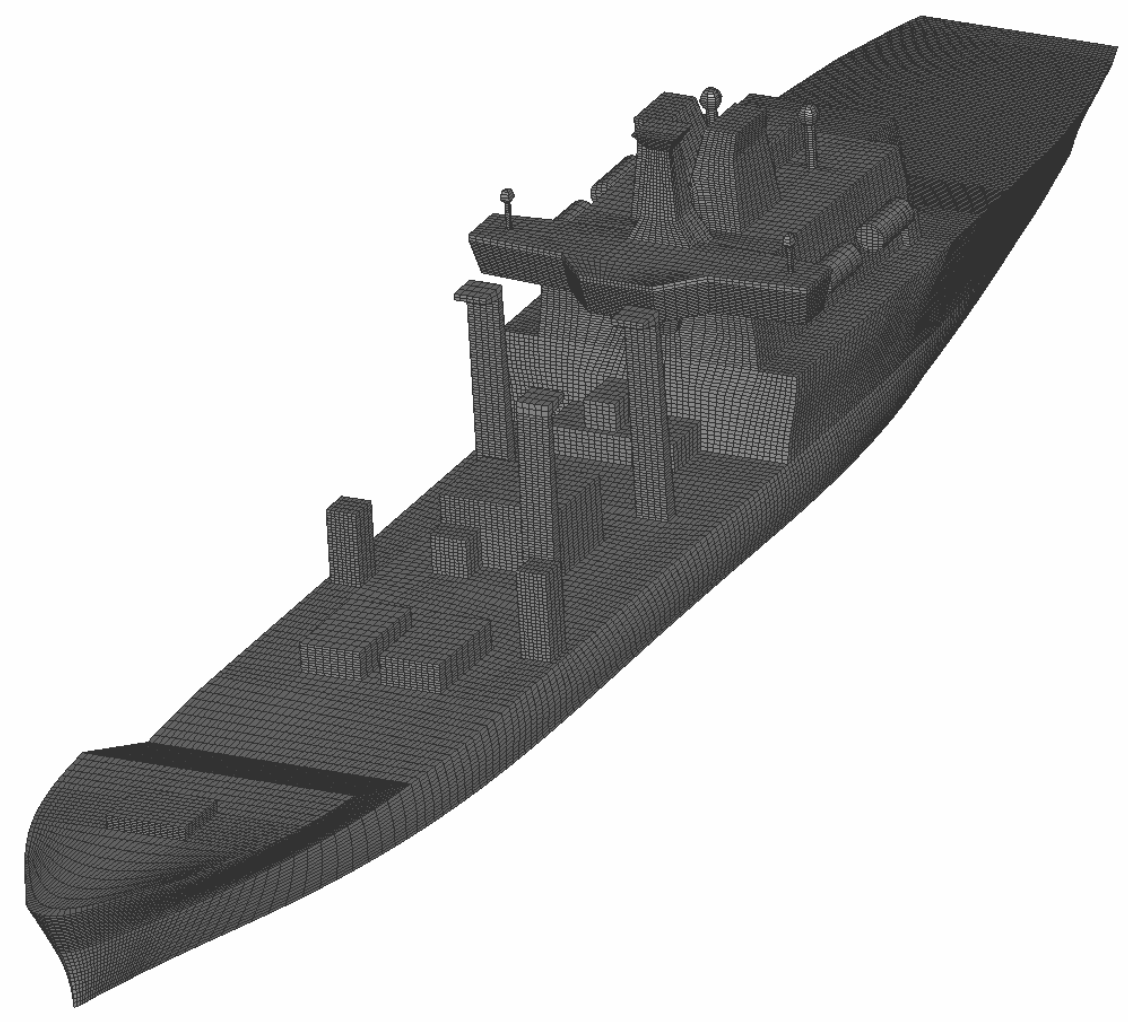

(b) Wave Class

Figure 2. Surface mesh for the two ships - note that the landing deck is at the rear of both ships 6 of 39 


\section{II.A.1. Type 23}

The mesh for the Type 23 consists of 72 individual blocks totalling 7.8 million points, which conform to the surface of the CAD model. Figure 2(a) shows the surface mesh for the Type 23, where the block boundaries have been omitted. The surface mesh was generated with even spacing for features not in close proximity to the landing deck, becoming gradually more refined towards the rear of the ship. Figure 2 (a) demonstrates that there is a good, smooth grid over the turret at the front of the ship and that the potential singularity at the bow has been removed by allowing the grid lines to 'flow' over the side of the deck. The bridge has been modelled accurately, as have the walkways/platforms along the side of the ship. Note that the spherical nature of the domes and turrets have been maintained (within the limits of grid resolution) and not reduced to square blocks.

There is grid refinement in the landing area located at the rear of the ship to capture as accurately as possible the large scale separation over the flight deck - the region over the flight deck consists of a single near-Cartesian block of 330,000 points in a region $25 \mathrm{~m} \times 25 \mathrm{~m} \times 25 \mathrm{~m}$. As this is the region where the intense shear layers generate a turbulent wake it was considered to be the area where grid refinement would have most impact. Previous experience suggests that grid cells which are approximately cubic in nature provide an even isotropic representation of turbulent fluctuations. ${ }^{47}$ The use of near-Cartesian grid geometry in the key areas of interest prevents the inevitable loss of accuracy incurred if highly skewed cells are present in the computational grid. In regions of poor grid quality the order of accuracy of the numerical method would be reduced to second order accuracy due to errors in defining the curvilinear transform.

\section{II.A.2. Wave Class AO}

The Wave Class AO is geometrically more complex to mesh than the Type 23. It required 121 blocks and 8.8 million points to reproduce geometry, which would affect the airwake structure.

Figure 2(b) shows the surface mesh, which illustrates the level of geometric detail captured in the meshing process. The lifeboats have been modelled as cylinders with the same volume as the original lifeboat. Additionally, there are features in the overhang underneath the bridge which could affect the development of any recirculation in that area which have been maintained. On the port side of the hangar there is a small recess which could affect the separation mechanism over the flight deck which has also been modelled. Again, care has been taken to refine the mesh over the landing deck (at the rear of the ship), where the grid has been generated with cells which are almost cubic and Cartesian, using over 400, 000 cells in the landing area alone. 


\section{II.B. Numerical Methods}

The Navier-Stokes equations are solved using the Godunov method. ${ }^{15}$ The problem of interest is split into a number of finite volumes where the initial values for the conserved variables are specified at the beginning of the simulation. The conserved variables are then evolved by computing the fluxes across the interfaces of each control volume.

Conventional LES, where an explicit subgrid model is added to the averaged NavierStokes equations, has been employed successfully in many prototype flows such as homogeneous decaying turbulence and turbulent channel flows, however it is known to provide excessive dissipation in flows where the growth of an initially small perturbation to fully turbulent flow must be resolved such as in flows which transition to turbulence. ${ }^{25,31}$ It has been recognised that some numerical schemes gain good results in complex flows without the explicit addition of a subgrid model. ${ }^{25}$ This occurs when the subgrid model is implicitly designed into the limiting method of the numerical scheme, based on the observation that an upwind numerical scheme can be rewritten as a central scheme plus a dissipative term. . $^{8} 11,18$ Such implicit subgrid models fall into the class of structural models, as there is no assumed form of the nature of the subgrid flow thus the subgrid model is entirely determined by the structure of the resolved flow. ${ }^{37}$

Using ILES, excellent results have been gained in simulation of flows as varied as RayleighTaylor and Richtmyer Meshkov instability, ${ }^{4,7,52-54}$ open cavity flow, ${ }^{8,20,45}$ free jets, ${ }^{2,17}$ channel flow, ${ }^{17}$ geophysical flows, ${ }^{26,41}$ delta wings ${ }^{16}$ and decaying turbulence. ${ }^{9,13,14,21,27,32,47}$

For this paper, the inter-cell numerical flux is computed based on the solution to the Riemann problem using the reconstructed variables at the left and right of the cell interface. For this step the HLLC approximate Riemann solver is employed as detailed in Toro. ${ }^{49}$ Higher order accuracy is achieved using MUSCL extrapolation, ${ }^{50}$ with the fifth-order limiter proposed by Kim and Kim. ${ }^{23}$

The standard MUSCL extrapolation has been augmented using the method of Thornber et al.,${ }^{48}$ which ensures uniform dissipation of kinetic energy in the limit of zero Mach number $(M)$. This modification extends the validity of the Godunov method to at least $M \approx 10^{-4}$, via a progressive central differencing of the velocity components, without changing the formulation of the underlying governing equations or sacrificing monotonicity of the density field. It was shown that the leading order kinetic energy dissipation rate is proportional to $u^{3} / \Delta x$, similar in form to that proposed by Kolmogorov ${ }^{24}$ for decaying turbulence ${ }^{48}$. It is this dissipation rate which acts as a subgrid model in the ILES framework, giving significantly improved high wavenumber performance compared to standard Godunov methods. ${ }^{45,48}$ This specific algorithm has been validated for use in turbulent flow fields through simulations of free shear layers separating from a sharp corner ${ }^{45}$ showing very good agreement with experiment, and various turbulent flow fields from homogeneous decaying turbulence through to 
shock induced multiple component turbulent mixing presented in Thornber et al. ${ }^{47,48}$

Finally, a second-order accurate three stage Runge-Kutta time-stepping method is employed, which due to an extended stability region (theoretical CFL limit of 2) enables a reduction in computational time. ${ }^{42}$

Each simulation ran for four days on twenty processors on the Cranfield High Performance Computer 'Astral' for the Wave Class AO, three days on twenty-four processors for the Type 23 Frigate.

\section{II.C. Initialisation of Flow Field and Boundary Conditions}

Two different initialisations were employed. For the SAIF project data the flow field was initialised using a mean atmospheric boundary layer,

$$
\begin{gathered}
u_{\text {cart }}=u_{0}\left(\frac{z}{200}\right)^{0.13} \cos \left(\theta_{w}\right), \\
v_{\text {cart }}=-u_{0}\left(\frac{z}{200}\right)^{0.13} \sin \left(\theta_{w}\right), \\
w_{\text {cart }}=0,
\end{gathered}
$$

where $u_{0}$ is the expected wind velocity at $200 \mathrm{~m}$ altitude, $u_{\text {cart }}$ is the velocity positive from bow to stern, $v_{\text {cart }}$ is positive from port to starboard, $w_{\text {cart }}$ is positive in the vertical direction, $\theta_{w}$ is the wind-over-deck (WOD) direction measured clockwise from the bow and $z$ is the altitude from sea level. The terminology $(.)_{\text {cart }}$ is used to distinguish these velocities from the ones measured experimentally - i.e. the 'along' wind velocity $u$, perpendicular to this in the horizontal plane the $v$ velocity, and the vertical velocity $w$.

For the comparisons against experiment there were several measurements of mean velocities and rms fluctuating velocities available from the experiments up to a scaled altitude of $80 \mathrm{~m}$. Below this altitude the flow field was initialised with a second order accurate interpolation of the experimental data onto the computational grid. Above $80 \mathrm{~m}$ altitude it was assumed that the boundary layer profile was a power law with an exponent of 0.13.

The numerical simulations require boundary conditions to complete the specification of the problem. Fixed inlet and extrapolated outlet boundary conditions were employed animations of the pressure and density field confirmed that there were no spurious oscillations generated. The upper surface was far field, taking constant flow field values at $z=200 \mathrm{~m}$. Following previous literature, the sea surface was modelled as a slip wall (flow perpendicular to the wall is set to zero, flow parallel can have a non-zero value) and the ship was modelled as a non-slip wall (all three velocity components are zero at the wall). Experience in cavity flow simulations have shown that even at very high Reynolds numbers the behaviour close to a sharp separation point can be wrongly predicted when using inviscid wall boundary 
conditions.

\section{II.D. Background Turbulence}

An important difference between the experimental results and numerical results is that in the wind tunnel there is a significant level of background turbulence whereas in the CFD simulations it is assumed that the background turbulence is negligible. This means that at the edge of the wake or outside the wake there will always be a discrepancy between LES and the experiment, regardless of numerical method. Thus although there is some disagreement between the LES and experiment in predictions of turbulent fluctuations outside the wake, this is not due to an error in the flow physics within the CFD simulation, simply a manifestation of the differences in initial atmospheric boundary layer conditions. Methods of introducing background turbulence into the LES simulations were investigated early in the project however they were deemed to be not sufficiently validated to employ in the framework of the current project.

The impact of not including background atmospheric turbulence into the simulation can be estimated by considering wind tunnel studies where the value of turbulent fluctuations in the incoming flow can be carefully controlled. Saathoff and Melbourne ${ }^{36}$ investigated separated flow past a bluff body. They noted that at higher values of background turbulence, the length of the separation bubble decreased by almost a factor of two as turbulent fluctuations increased from $\sigma_{u} / U=0$ to $\sigma_{u} / U \approx 0.1$. Above this value the length of the separation decreases only marginally for higher turbulence levels. The assumption of negligible incoming turbulent fluctuations can thus have a strong influence on the final solution, but is a common assumption in unsteady simulations of ship airwakes when using LES methods. As the computational grid is significantly stretched at the inlet, any low level fluctuations added to the flow at the inlet would most likely dissipate before reaching the ship.

\section{II.E. Convergence of Average Flow Properties}

It is well known that it can take a significant period of time to achieve accurate, converged time-averaged statistics when the mean flow is turbulent. The data measured in these simulations span a total of forty seconds. Figure 3 shows the time traces of $u, v$ and $w$ velocities over the forty second period for a single measurement point in the flight path.

From the initial time traces it was decided that a reasonable time to start the averaging was after the flow had crossed the full length of the ship two times - equating to a time of approximately 10 seconds. It can be seen that this is a reasonable assumption as the flow appears reasonably steady after approximately 5 seconds - note the large fluctuation at 3.5 seconds due to a 'start-up' vortex crossing the flight deck. Figure 3 demonstrates the 


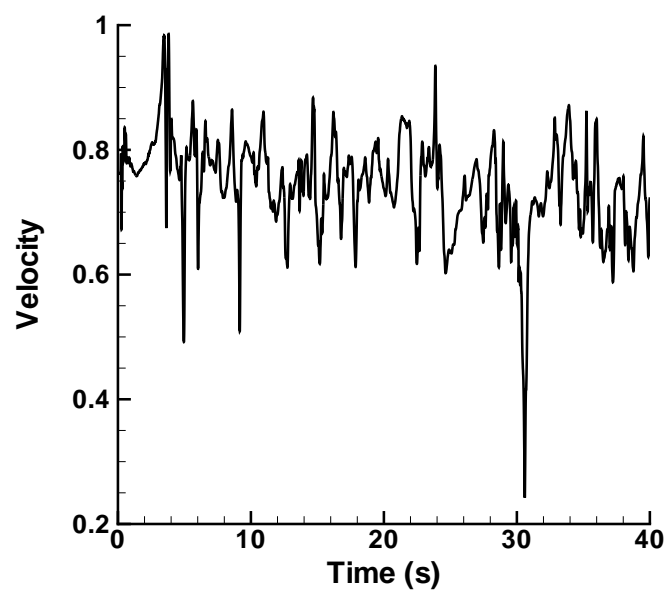

(a) $u$ velocity

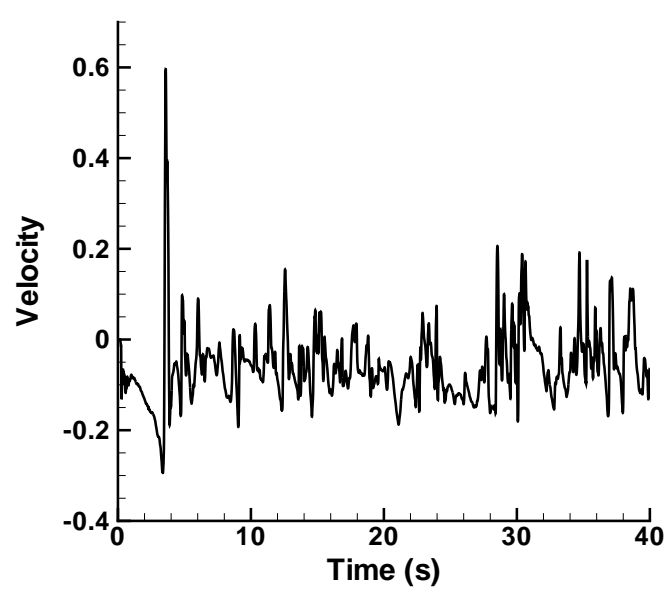

(c) $v$ velocity

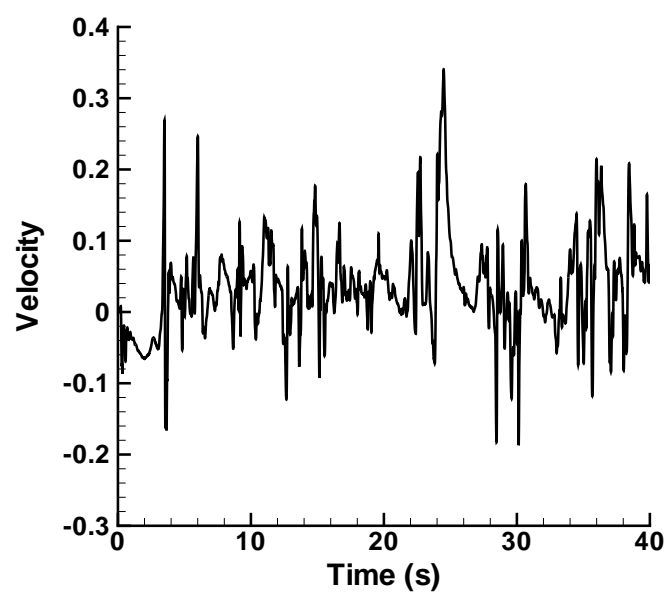

(e) $w$ velocity

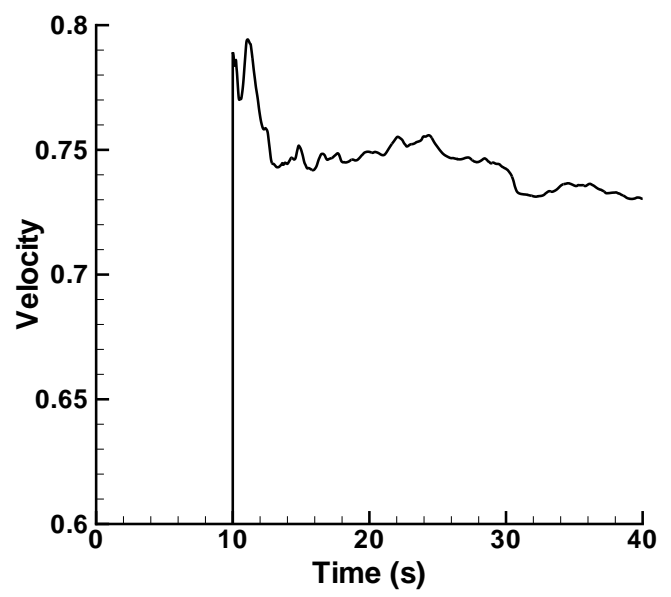

(b) time averaged $u$

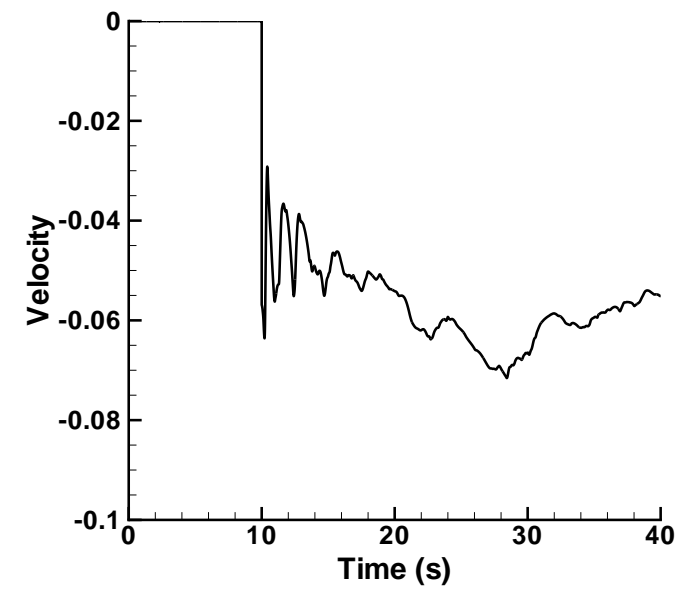

(d) time averaged $v$

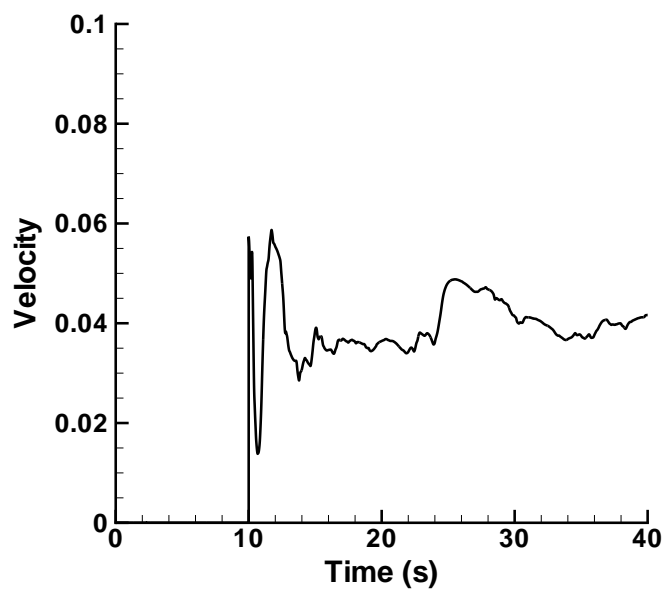

(f) time averaged $w$

Figure 3. Instantaneous velocity measurements at a single point in the flight path for the Wave Class AO at $30^{\circ}$ WOD (left) and time averaged quantities (right). Note the $u$-direction velocity is in the 'along' wind direction, $v$ is perpendicular to this and $w$ is vertical. 
difficulty in converging such a noisy turbulent signal, even after 30 seconds of averaging there is still some small fluctuations in all three flow quantities. Additionally, there are single time intermittency events such as that which occurs in the $u$ velocity at about 30 seconds - due to the passage of an unusually strong vortex over the flight deck.

Examining the experimental data shows that there is significant turbulent kinetic energy in modes lower than that captured in this simulation, i.e. frequencies lower than $1 / 30 \mathrm{~Hz}$ (when WOD scaled). Such a long time mean velocity fluctuation (equivalent of one oscillation every 15 crossings of the air over the length of the ship) would not be captured here and hence would influence the mean flow values not the turbulent velocity. It is not clear if these ultra-low frequency events are due purely to the ship or to ship-wind tunnel interaction.

Taking these considerations into account it can be concluded that the mean flow quantities are reasonably converged to within approximately $\pm 10 \%$.

The results in this section are plotted as plots of 'along wind velocity' $u$, 'cross-wind' velocity $v$ and vertical wind velocity $w$. The fluctuating velocities are defined as

$$
u^{\prime}=\sqrt{\overline{(u-\bar{u})^{2}}}
$$

where (.) indicates a time averaged quantity. The fluctuating velocities are output every computational time step, equivalent to a resolution of $100 \mathrm{~Hz}$ for each point in the array and then post processed to gain the required quantities.

\section{Type 23 Frigate}

\section{III.A. Flow Phenomenology}

In this section the qualitative nature of the flow field will be discussed, such as how the atmospheric boundary layer interacts with the ship, where the major separations occur and whether these propagate into the standard approach path or not. Figures 4 and 5 show visualisations of isosurfaces of $Q$ for the Type $23,{ }^{22}$ where

$$
Q=-\frac{1}{2} \frac{\partial u_{i}}{\partial x_{j}} \frac{\partial u_{j}}{\partial x_{i}}
$$

On each subfigure the major flow features are discussed and labelled as 'a, b, c, ..' and so on. Note that although results were generated for $0^{\circ}, \pm 15^{\circ}, \pm 30^{\circ}, \pm 45^{\circ}, \pm 60^{\circ}, \pm 90^{\circ}$, $\pm 135^{\circ}, 180^{\circ}$ we only present one half of the data produced as the results are qualitatively approximately symmetric for symmetric wind conditions. 


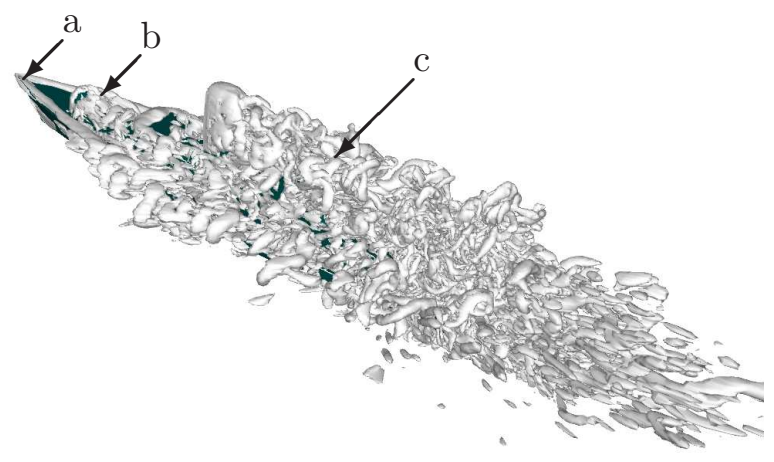

(b) $15^{\circ}$

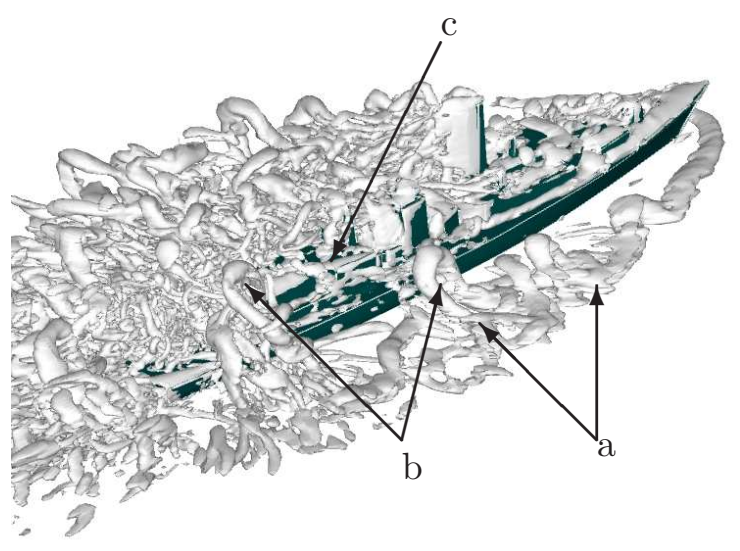

(c) $30^{\circ}$

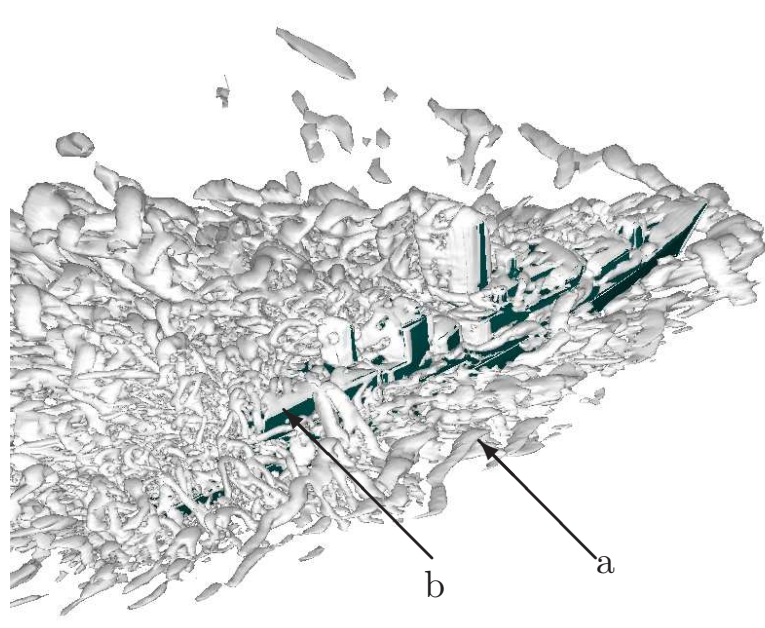

(e) $60^{\circ}$

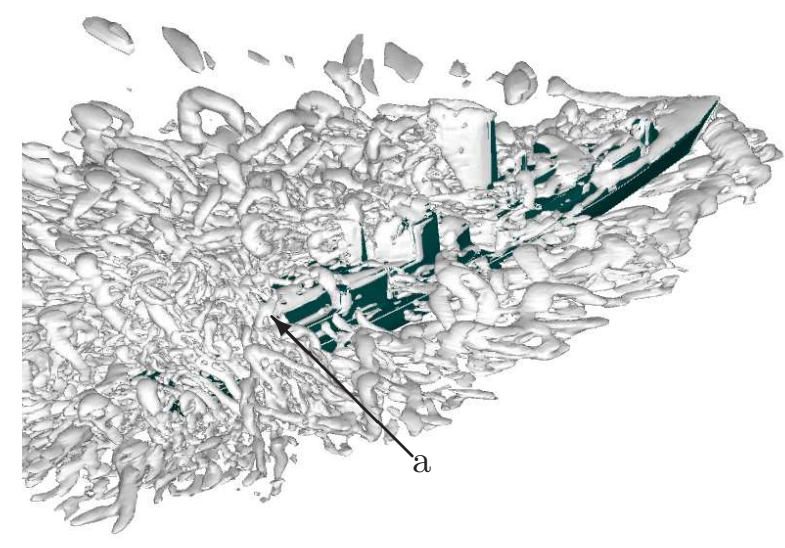

(d) $45^{\circ}$

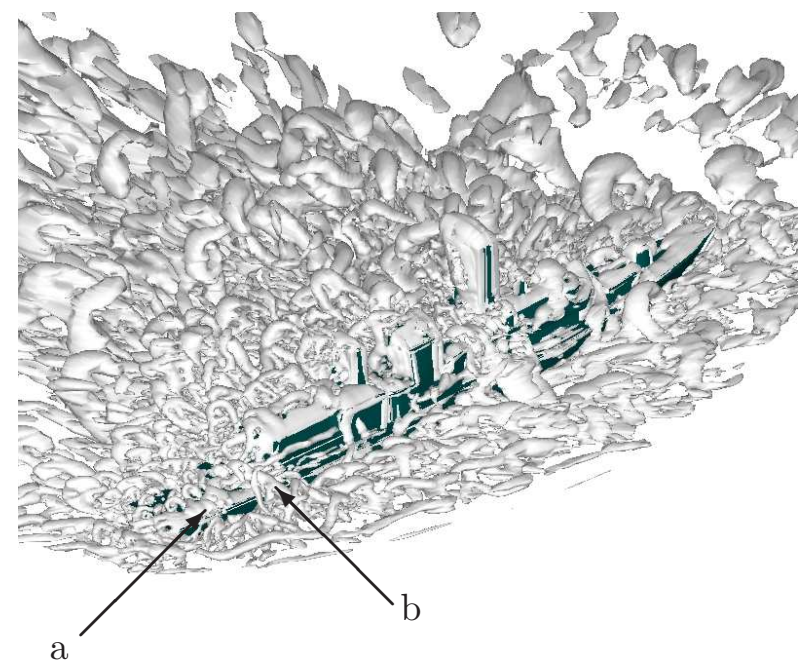

(f) $90^{\circ}$

Figure 4. Visualisations of isosurfaces of $Q$ illustrating the flow features at WOD angles $0^{\circ}$ to $90^{\circ}$ for the Type 23 


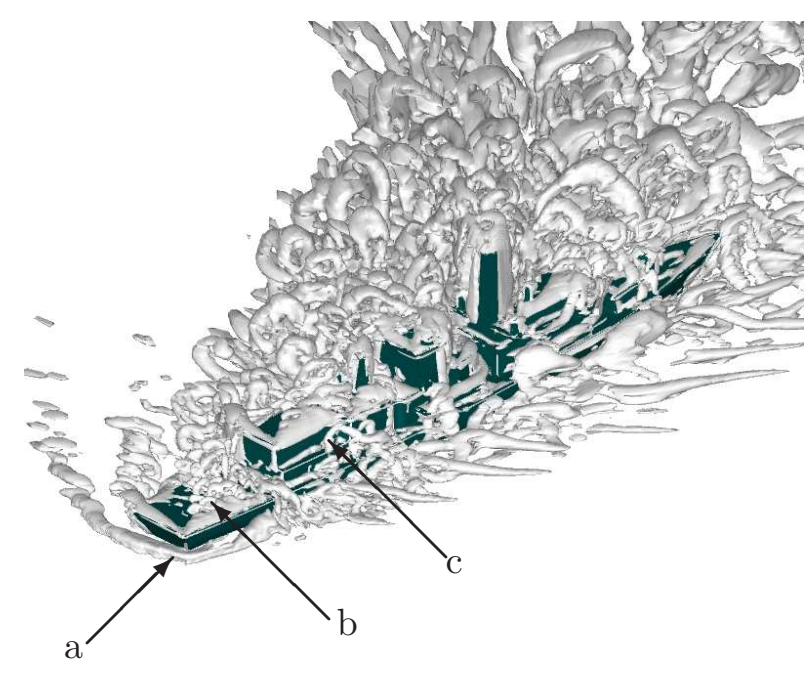

(a) $135^{\circ}$

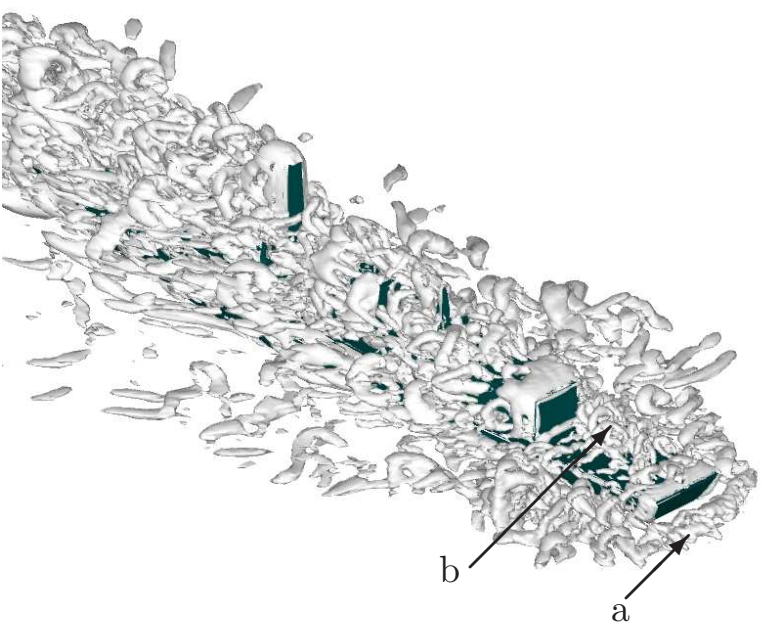

(b) $180^{\circ}$

Figure 5. Visualisations of isosurfaces of $Q$ illustrating the flow features at WOD angles $135^{\circ}$ to $180^{\circ}$

III.A.1. $0^{\circ}$

There are two coherent vortices on either side of the bow of the Type 23 (beginning at point ' $a$ '), which break down after their interaction with the wake from the turret. Interestingly, the wake from the turret (labelled as 'b') passes past the port side of the mast, however, as the structure is geometrically symmetric, it could just as easily pass over the starboard side in reality - this will be strongly dependent on the exact angle of the incoming flow slightly starboard and it will pass on the port side and vice versa. There is an interaction of this vortex with the bridge and a small region of separation there. The greatest influence on the airwake around the flight deck comes from the mast, the twin radomes, the vent/uptake structure, and the separation over the top of the hangar highlighted as region 'c'. This separation leads to a recirculation zone encompassing about $40 \%$ of the flight deck length, at a height of less than $6 \mathrm{~m}$. The wake from the upstream structures passes over this recirculation zone and hence forms the airwake which the helicopter would fly through.

\section{III.A.2. $15^{\circ}$}

At this angle there is a strong influence of the platforms to either side of the bridge structure on the generation of longitudinal vortices (structure 'a') along the full starboard length of the ship, interrupted only briefly where there are gaps in the superstructure between, for example, the mast and the vent/uptake. The vortex eventually becomes large enough to pass over the top of the starboard side of the hangar (point ' $b$ '), where it merges with a vortex generated along the upper edge of the starboard side of the hangar which has the same sign of vorticity (rotating in the same direction). The wake has a clear counter-clockwise rotation 
(as viewed facing the bow of the ship) which would lead to downdrafts on the port side of the wake and updrafts above the flight deck.

\section{III.A.3. $30^{\circ}$}

There is a stronger influence of the large turbulent vortex 'a' off the starboard side of the ship, which periodically ejects smaller (but still substantial, e.g. structure 'b') vortices over the structure of the ship. Closer to the flight deck, the recess along the starboard side of the hangar (the clear asymmetry present on the starboard side of the hangar labelled as 'c' in Figure 5 (a)) causes a small longitudinal vortex to be generated, rotating within the recess, finally exiting above the flight deck. A second vortex is located along the top edge of the starboard side of the hangar, which is amplified in diameter as the vortex passes over the fore part of the recess (point ' $c$ '). As with the $15^{\circ}$ case, the airwake has a clear counter-clockwise rotation.

\section{III.A.4. $45^{\circ}$}

The structures present at $45^{\circ}$ are very similar to those at $30^{\circ}$, except that at this angle it is unlikely that the standard approach path will take the helicopter into any region of the airwake substantially influenced by the ship structure in front of the hangar. The principal source of turbulence is the separated flow from the starboard side of the hangar (point ' $a$ '), primarily separating at the corner where the top of the hanger meets the recess and at the rear of the hanger. The vortices shed from the rear starboard side of the hangar cross the centre of the flight deck generating a strong shear flow between relatively quiescent flow (not many turbulent fluctuations) and the airwake. Interestingly, the flow sticks to the downwind side of the superstructure in a similar manner to the suction side of an airfoil, before joining the recirculation zone at the port edge of the hangar.

\section{III.A.5. $60^{\circ}$}

At $60^{\circ}$ there is a greater influence of the turbulent vortex which lies to the starboard side of the ship, between the hull and the sea (structure 'a'). Smaller vortices (still on the order of $2 \mathrm{~m}$ in diameter) are shed from this vortex and pass over the aft of the ship. Vortices generated from the upper starboard edge of the hangar (location 'b') tend to pass over the hangar, instead of travelling along the hangar then passing over the flight deck as they did at angles below $60^{\circ}$. Examining the airwake as a whole the large flow structures all emanate from the ships superstructure, especially the bridge, mast and vent/uptake - this is common to all flow angles. 
The incident flow onto the flight deck forms a shear layer which rolls up, becoming turbulent (region 'a'), but remaining relatively low to the deck $(\leq 6 m)$. Larger structures are periodically shed over the top of this shear layer by the vortex which lies off the starboard side of the ship (flow structure ' $b$ '). As for the $60^{\circ}$ case, there are separated regions of flow on the top and aft of the hangar, however these are localised and do not cross the standard port approach path - the principal influence for a port approach is the shear layer from the deck edge and vortices shed from the off-starboard (between hull and sea) vortex.

\section{III.A. ${ }^{7} .135^{\circ}$}

At $135^{\circ}$ there is a horseshoe vortex sitting just to the aft starboard corner of the ship (feature ' $a$ '). The wind passes over this vortex, then separates at the aft starboard corner, generating a shear layer (labelled 'b'). This shear layer becomes turbulent and is accelerated across the flight deck, passing past the port side of the hangar. A similar process occurs at the starboard aft corner of the hangar, generating a turbulent shear layer. The structure of the flow field at the leading corner of the flight deck and the hangar is very similar to that reported by Castro and Robins ${ }^{3}$ for a surface mounted cube at a $45^{\circ}$ angle of incidence.

\section{III.A.8. $180^{\circ}$}

The flow close to the flight deck consists of three mutually interacting phenomena. There is a rotating horseshoe vortex just behind the ship ('a') and this deflects the mean flow upwards, striking against the aft of the ship. This generates a shear layer with hairpin structures, which impacts against the hangar door (in region 'b'). As these structures impact against the door they either pass over the top of the hangar, or they are sucked into a recirculation vortex (another horseshoe vortex) which is wrapped around the base of the hangar door. There is further separation from the edges of the hangar and usual large scale vortex streets from the superstructure located further forwards.

\section{III.B. Comparison with Experimental Data}

This section compares the CFD results with two different sources of experimental data provided by Qinetiq. ${ }^{33}$ The first data source are wind tunnel measurements taken in an atmospheric boundary layer tunnel. Two data sets were provided for mean and fluctuating longitudinal velocities from the wind tunnel experiments allowing an evaluation of the uncertainty in the measured data. In addition to these results, comparison will also be made to data measured on board a full scale Type 23 at sea. These two data sources are referred to in figure legends as 'Wind Tunnel' and 'Fullscale' respectively. 


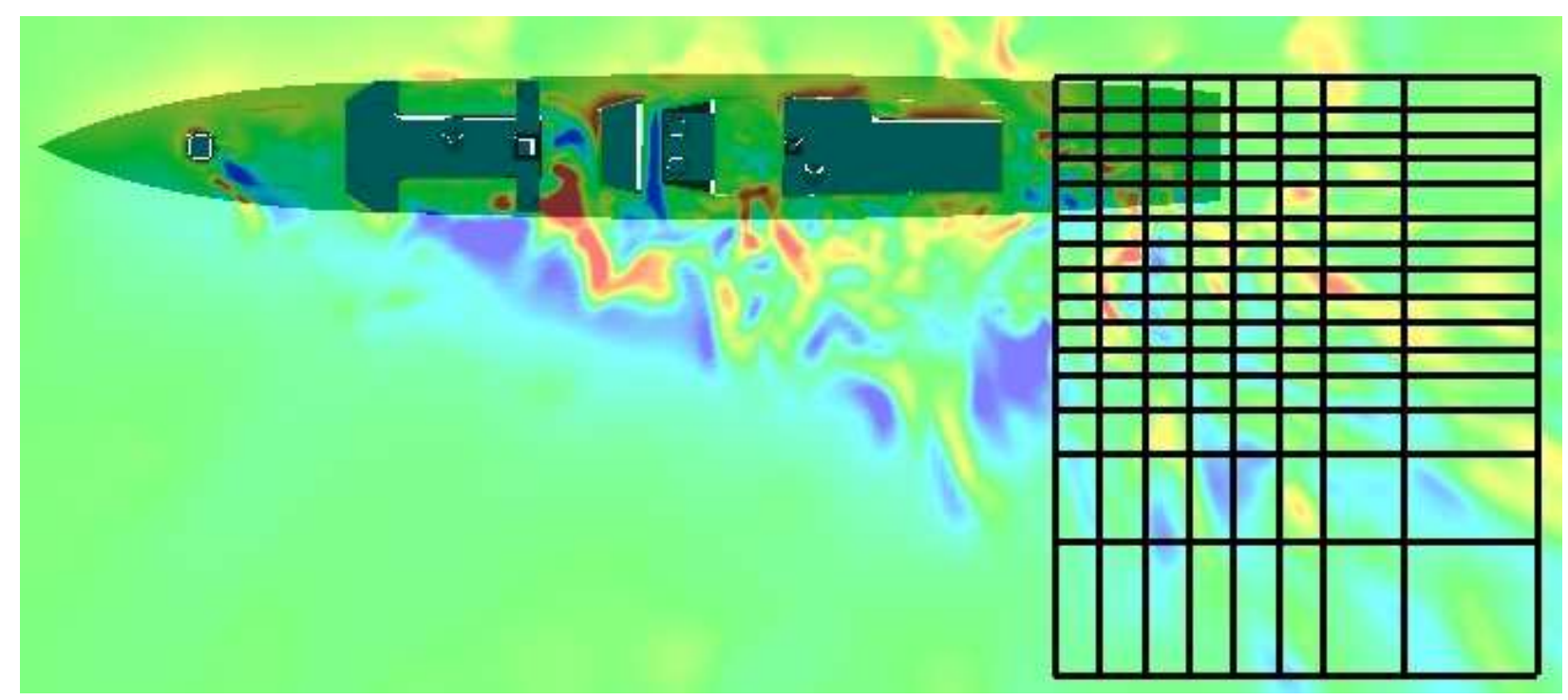

Figure 6. Position of the array of data points sampled in the LES simulation for comparison with experiment. Contour flood shows the instantaneous vertical velocity component at $z=6 \mathbf{m}$

To compare the ILES results with wind tunnel experiment an array of 576 data points were extracted at each of $3 \mathrm{~m}, 6 \mathrm{~m}, 9 \mathrm{~m}$ and $12 \mathrm{~m}$ height above the flight deck. The location of the array is shown schematically in Fig. 6 overlaid on top of an instantaneous snapshot of vertical velocity magnitude. A comparison was made for Green $30^{\circ}$ (i.e. a wind coming from the starboard direction at $30^{\circ}$ from full ahead) and Green $90^{\circ}$ winds however this paper will present only results for Green $30^{\circ}$. Results of an equivalent standard were achieved for the $90^{\circ}$ case. These two cases were chosen as previous simulations with RANS methods have shown them to be the most difficult to capture accurately.

As the velocity was reduced to 30 knots in the simulations comparing with experiment the amount of physical time simulated was extended to 67 seconds giving 50 seconds physical sampling time in total. The experimental data was averaged for over 4000 seconds of physical time.

In the LES, the results are taken from the nearest grid point in the computational mesh to that measured in experiment - it was verified that this was within $0.2 \mathrm{~m}$ of the specified point close to the flight deck and $1.7 \mathrm{~m}$ at the furthest points away (lower right hand corner in Fig. 6).

Three sets of data are presented showing the typical agreement gained (Fig. 7), good agreement (Fig. 8) and the worst agreement (Fig. 9), each plotting a single $y$ line from the experimental grid, where the origin for $y$ is at the centreline of the ship. Comparing the mean flow velocities between the experiments and LES show an excellent overall agreement. The best agreement is at the data points close to the flight deck where the mesh is finest. Poorest agreement is in Fig. 9, however even this is still a reasonable match to experimental results. 


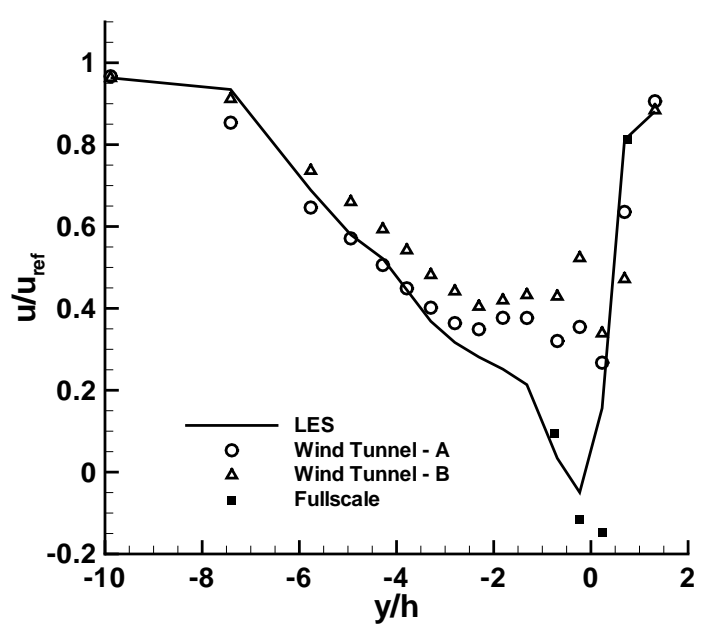

(a) u

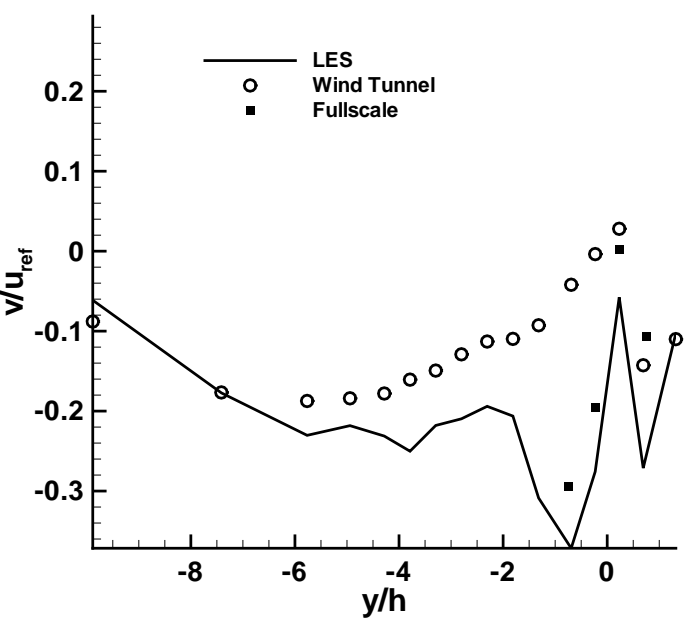

(c) $\mathrm{v}$

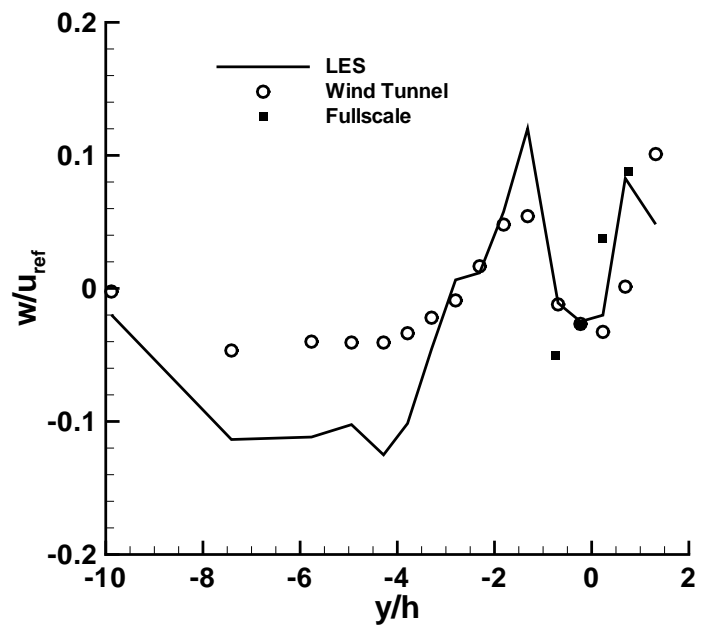

(e) $\mathrm{w}$

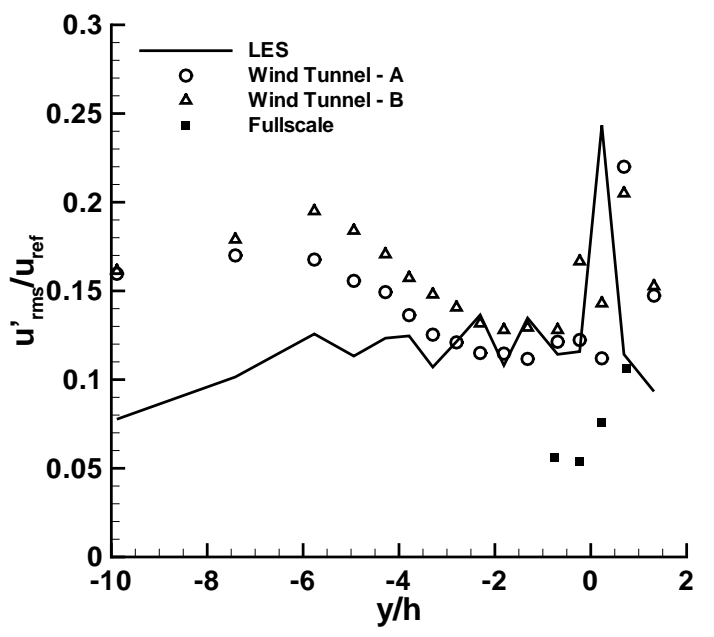

(b) u'

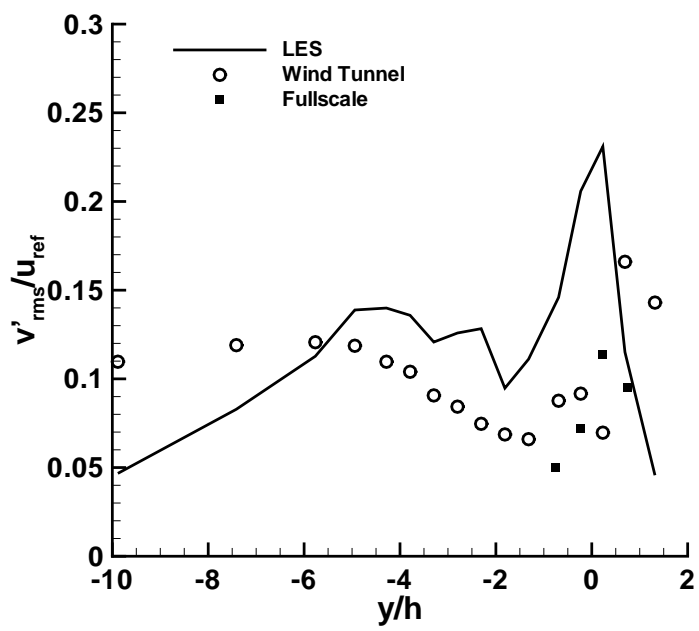

(d) v'

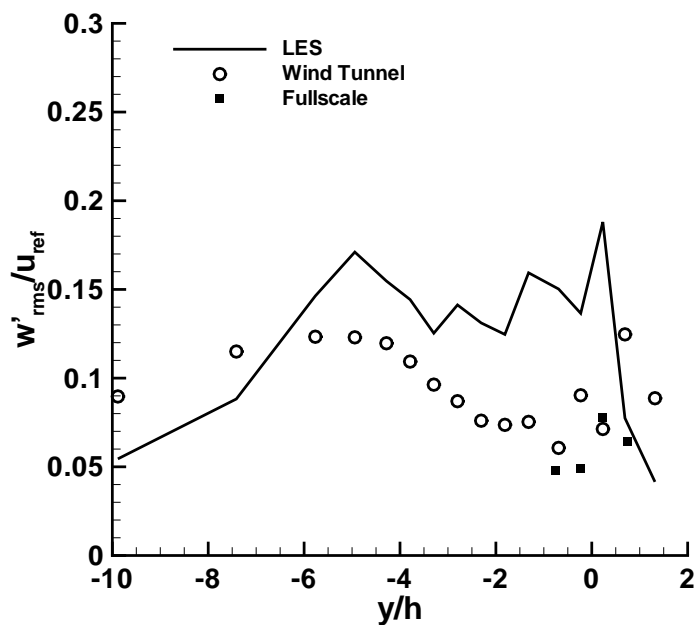

(f) w'

Figure 7. 'Typical' agreement compared to experimental data. Flow properties for the line at $x=5.9 m, z=3 m$ for the Type 23 


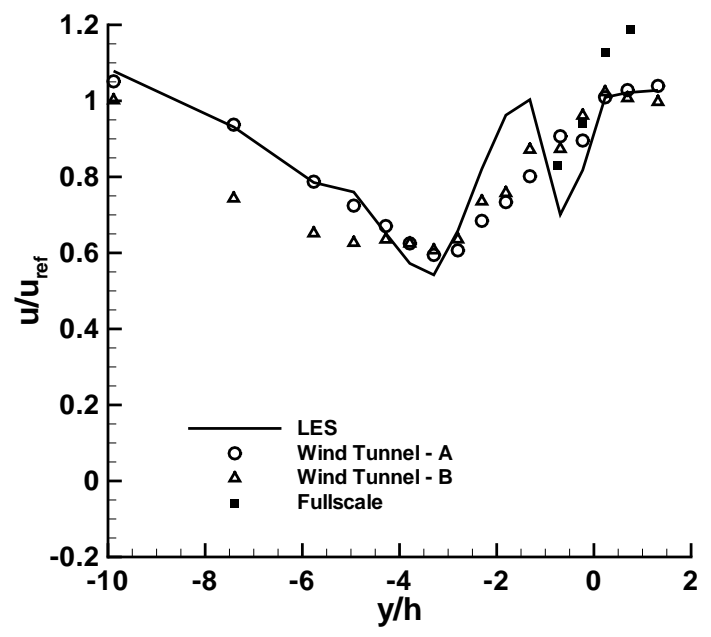

(a) u

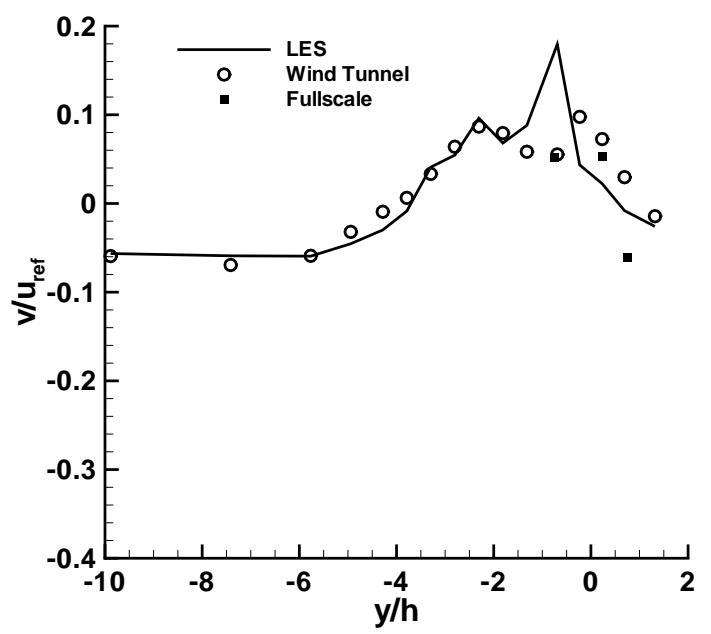

(c) $\mathrm{v}$

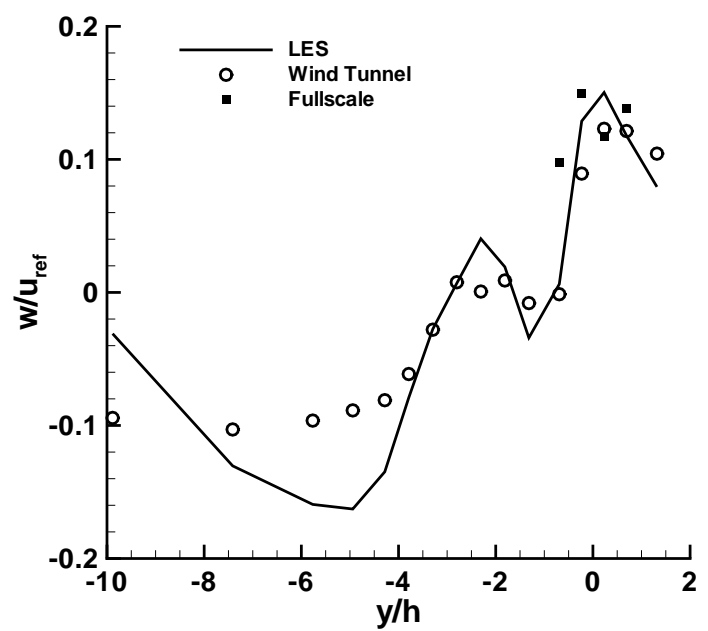

(e) W

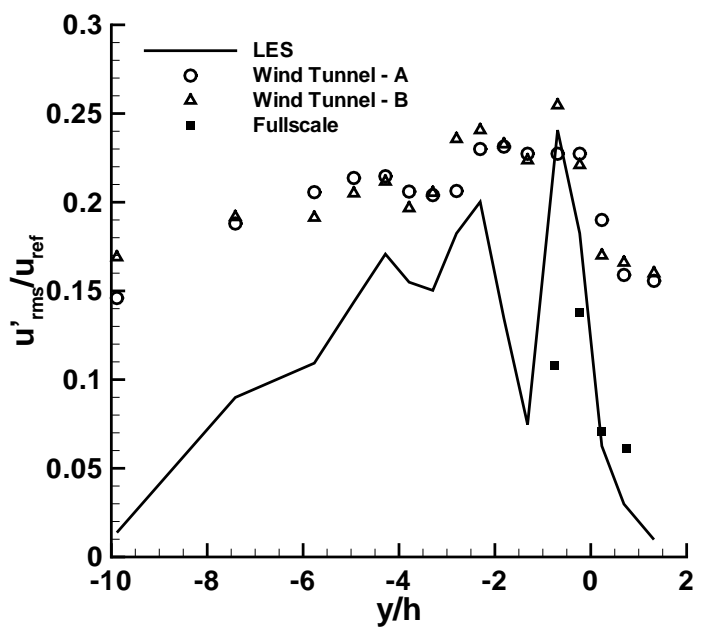

(b) u'

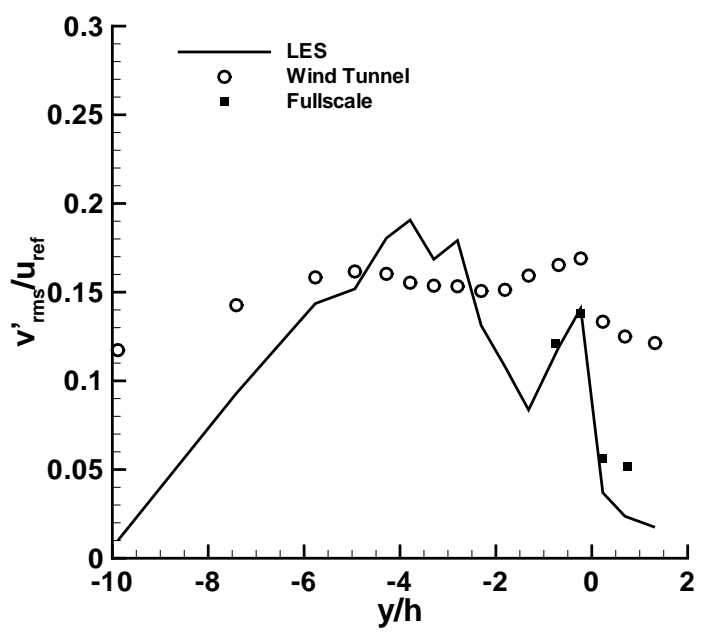

(d) v'

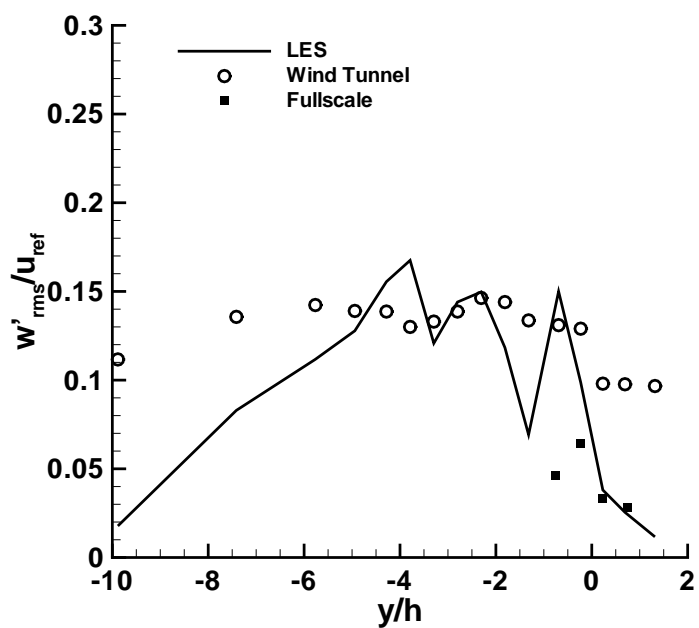

(f) w'

Figure 8. 'Good' agreement compared to experimental data. Flow properties for the line at $x=5.9 m, z=9 m$ for the Type 23 


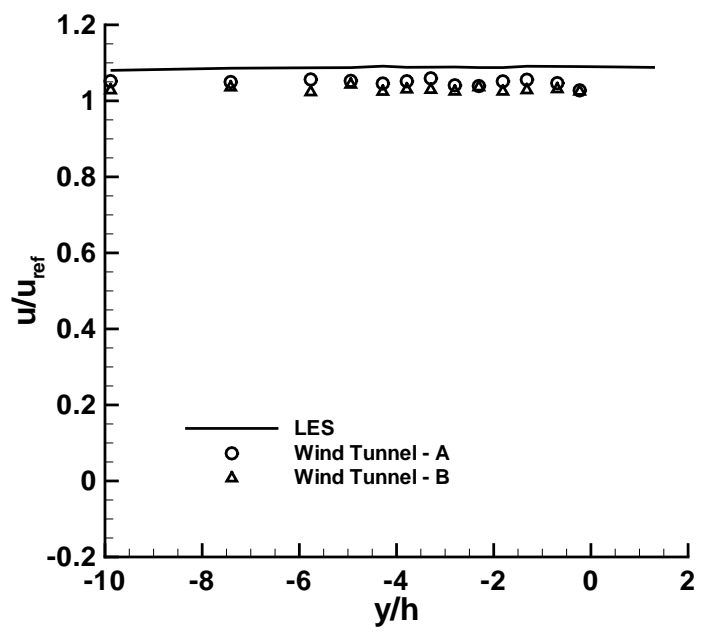

(a) u

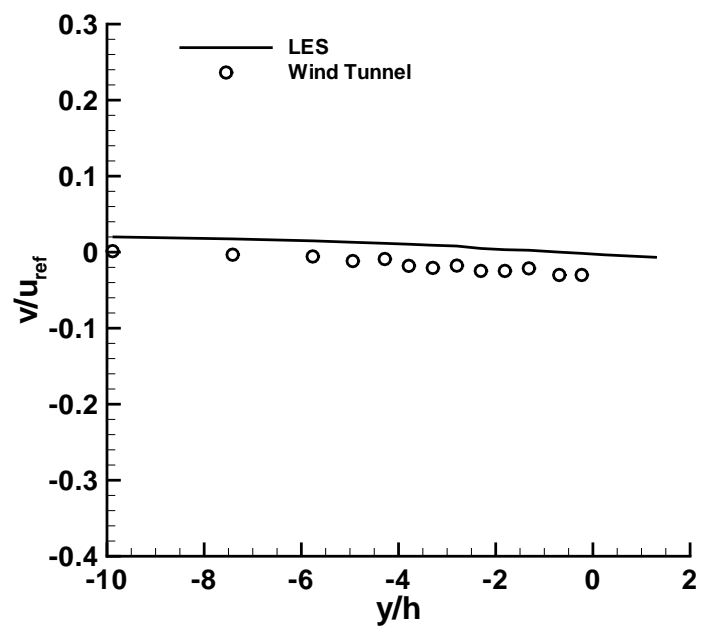

(c) $\mathrm{v}$

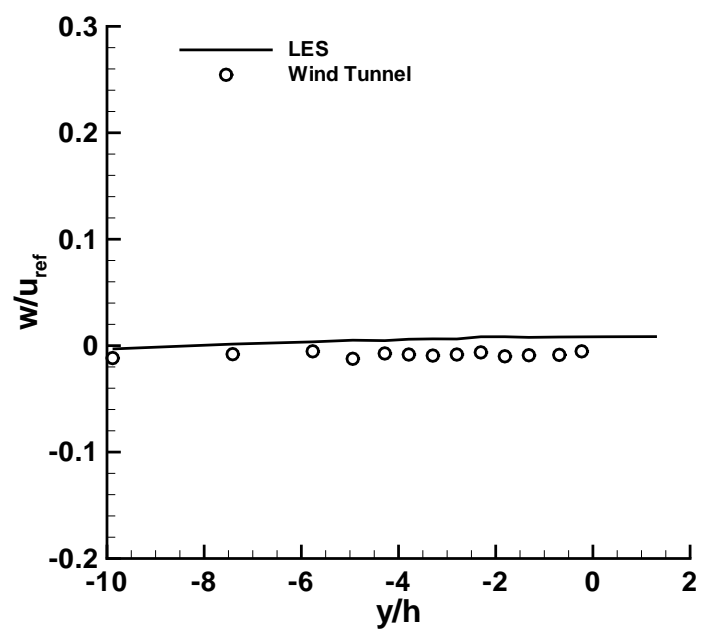

(e) w

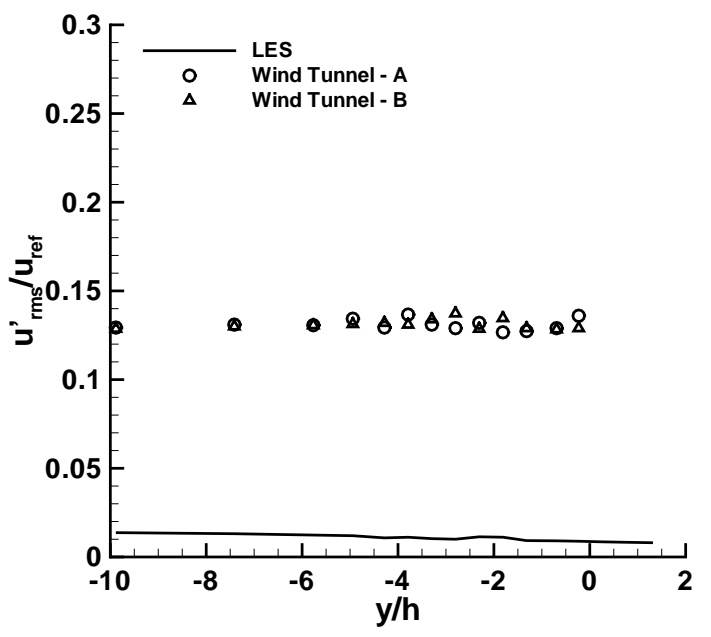

(b) u'

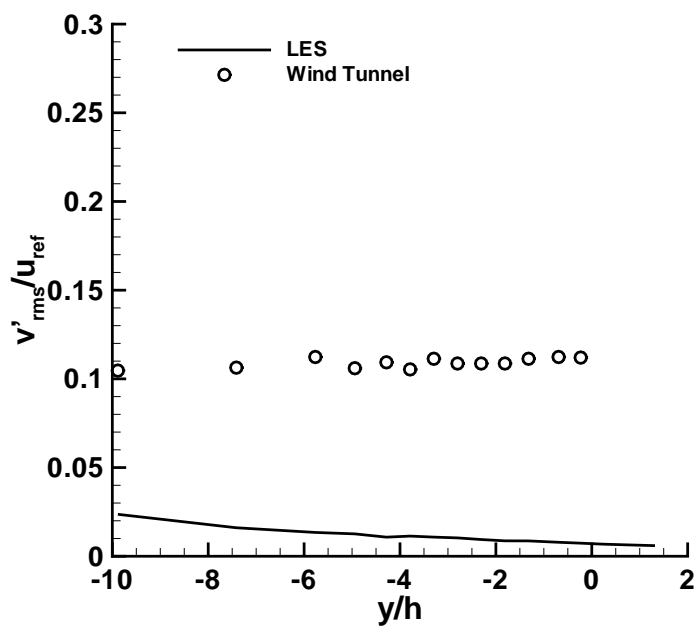

(d) v'

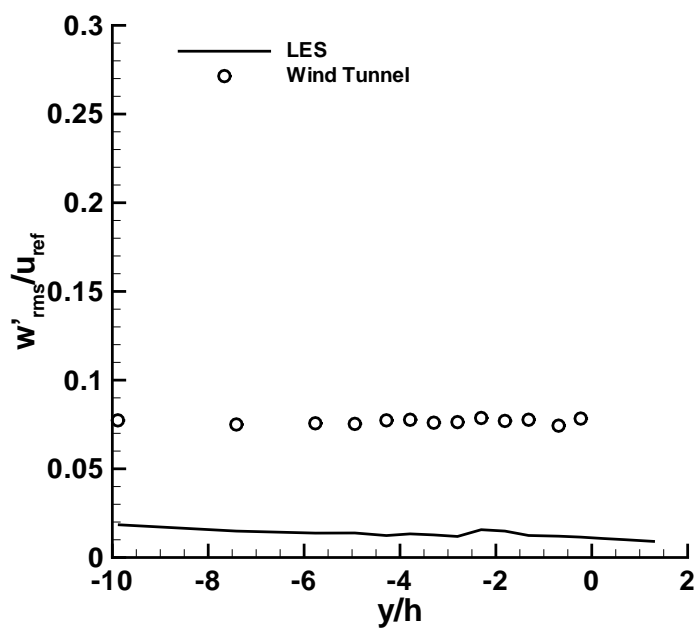

(f) w'

Figure 9. 'Worst' agreement compared to experimental data. Flow properties for the line at $x=60 \mathrm{~m}, z=12 \mathrm{~m}$ for the Type 23 
The fluctuating velocities are more difficult to capture. The key issue in interpreting the plots is that the wind tunnel has a high level of background turbulence of unspecified spectrum which was not included in the LES as discussed in Section II.D. This means that outside the wake the LES will tend to zero turbulent fluctuations whereas the experimental results tend towards the freestream value. Hence the ideal expected results would be a match in the regions within the airwake, however not outside the wake. This explains a poor match in Fig. 9, where these co-ordinates lie outside the airwake as illustrated in Fig. 6 a).

The full scale results also have lower atmospheric turbulent kinetic energy as can be seen in Figs. 8 (b) (d) and (f) for $y>0$. The CFD data follows the full scale data closely over the flight deck, where the level of turbulent kinetic energy drops below that of the background kinetic energy in the wind tunnel experiments. In general there is a good qualitative and quantitative agreement within the airwake, the CFD giving a slight overestimation of fluctuating velocities over the flight deck.

The Power Spectrum Densities (PSD) have been calculated using the raw data provided for use on the SAIF project by SEA above the landing spot. The PSD is defined as the power in the fluctuating turbulent velocity in a given direction. This is computed as the square of the Fourier transform of the flow velocity, divided by the time duration of the time sample, giving an energy per unit time per frequency band or Power Spectral Density. To give consistency between the methods, the PSD for the experimental data was computing using the same software as that used for the LES data.

Figure 10 shows the spectra of the three velocity components. To aid interpretation lines proportional to Frequency $^{-5 / 3}$ and Frequency ${ }^{-4}$ have been drawn on each figure. The first is the theoretical power law dependency of kinetic energy as a function of frequency, ${ }^{24}$ the second power law appears in the experimental results at frequencies higher than about $3 \mathrm{~Hz}$.

The lowest frequency in the numerical results is at $0.02 \mathrm{~Hz}$, hence at frequencies below $0.1 \mathrm{~Hz}$ one would not expect very good results as the PSD represents the average behaviour of only one to five full periods in the given simulation time frame. Despite this, at frequencies between $0.1 \mathrm{~Hz}$ to $10 \mathrm{~Hz}$ there is excellent agreement with the experimental data in all three velocity components. The LES results are more oscillatory, indicating either that there are certain unstable modes (possibly related to a given shedding frequency) or simply that the averaging period is not sufficient to give a smooth curve.

Of great interest is the observation that both the numerical and experimental results have a 'kink' in the power spectrum at $3 \mathrm{~Hz}$, where it changes from a $F^{-5 / 3}$ behaviour to a $F^{-4}$ behaviour. This indicates that above $3 \mathrm{~Hz}$ the amount of energy in each mode falls dramatically. The mean velocity of a vortex at a given frequency $F^{\prime}$ is given by 


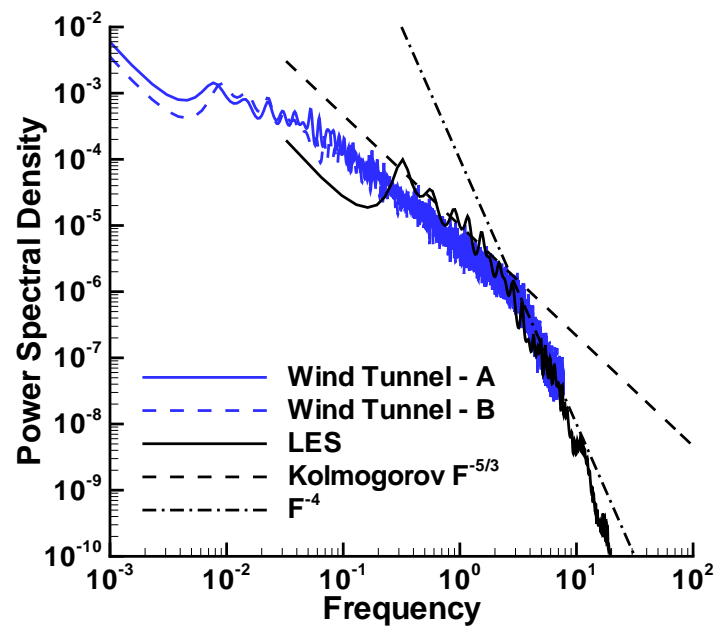

(a) u velocity $30^{\circ} \mathrm{WOD}$

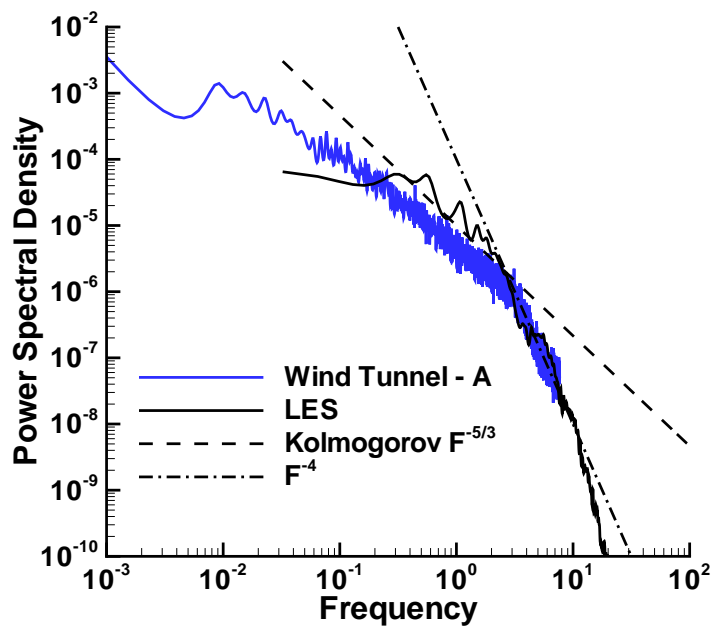

(b) v velocity $30^{\circ} \mathrm{WOD}$

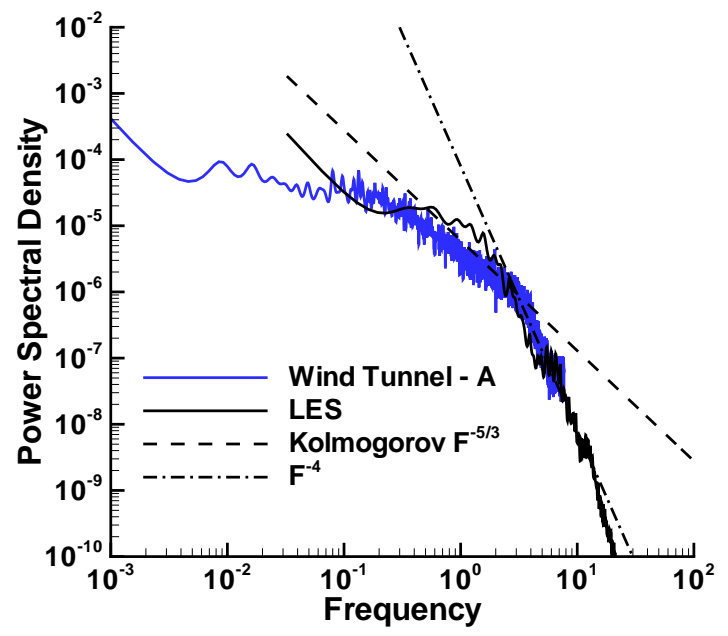

(c) w velocity $30^{\circ} \mathrm{WOD}$

Figure 10. Turbulent power spectra for the $\mathrm{u}, \mathrm{v}$ and $\mathrm{w}$ velocity components compared to experiment at $30^{\circ}$ WOD for the Type 23 Frigate. 


$$
u^{\prime 2} \propto \int_{F^{\prime} / 2}^{F^{\prime}} P(F) d F^{\prime},
$$

meaning that $u^{\prime} \propto F^{\prime-3 / 2}$ for frequencies above $\approx 3 \mathrm{~Hz}$, whereas for the frequency range below $3 \mathrm{~Hz}$ the velocities scale as $F^{\prime-1 / 3}$. This result implies that frequencies significantly higher than $3 \mathrm{~Hz}$ will have very small or negligible velocities. If such an observation is general then this will make the problem of airwake prediction more amenable to Large Eddy Simulation than previously considered.

\section{Wave Class AO}

\section{IV.A. Flow Phenomenology}

Figures 11 and 12 show visualisations of isosurfaces of $Q$ for the Wave Class AO, which illustrate the flow phenomenology. Again, the flow is qualitatively symmetric for symmetric wind conditions, so only one half of the WOD directions simulated are discussed.

IV.A.1. $0^{\circ}$

At $0^{\circ}$ the flow consists of two long vortices attached to either side of the bow of the ship (labelled ' $a$ ') and vortex shedding off all of the main structural features such as the masts and radomes. The wake from the central structure of the ship separates over the top of the hangar door, generating a turbulent recirculation zone over the flight deck. The influence of the lifeboats on the flow around the flow close to the flight deck appears to be a broadening the wake and an injection of turbulence into the higher speed flow which passes around the bridge area (feature 'b').

\section{IV.A.2. $15^{\circ}$}

At this angle of attack the key features influencing the flow around the flight deck are the large vortex attached to the starboard side of the bow (features 'a'), which becomes turbulent generating substantial wind fluctuations and a secondary wake generated off the starboard side of the bridge where it projects in front of the lifeboats (location 'b'). This wake is swept over the flight deck by the action of the mean flow. The final contribution to the flow are the lifeboats themselves. A region of accelerated flow underneath the lifeboats forms a longitudinal vortex (relative to the ship - highlighted at point 'c') which becomes turbulent and expands rapidly over the edge of the flight deck - turbulence shed from the boats themselves passes over the deck at an altitude of $5-10 \mathrm{~m}$. During a port approach the helicopter will be influenced also by the wake generated by the large scale structures such as 


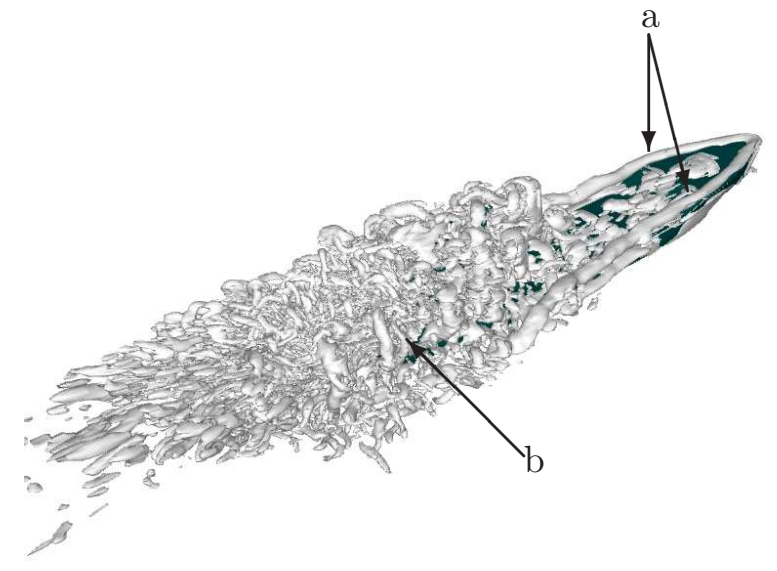

(a) $0^{\circ}$

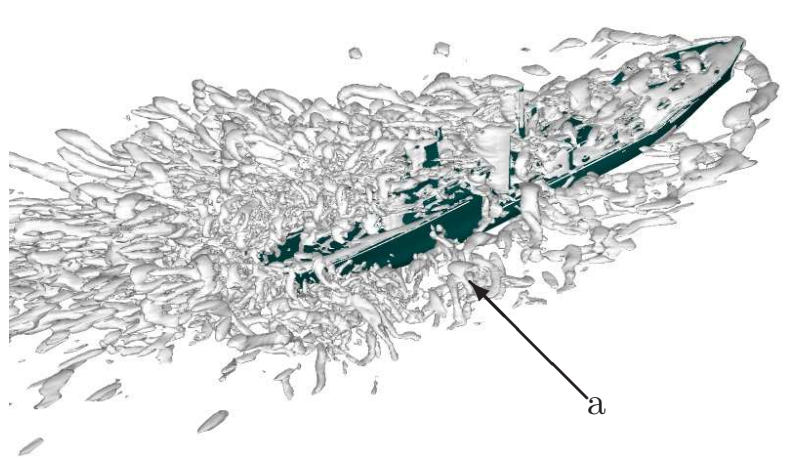

(c) $30^{\circ}$

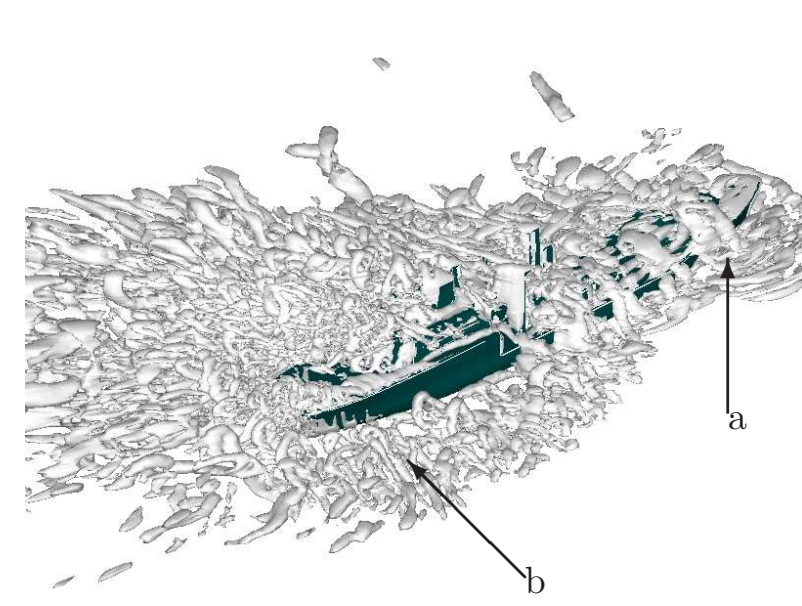

(e) $60^{\circ}$

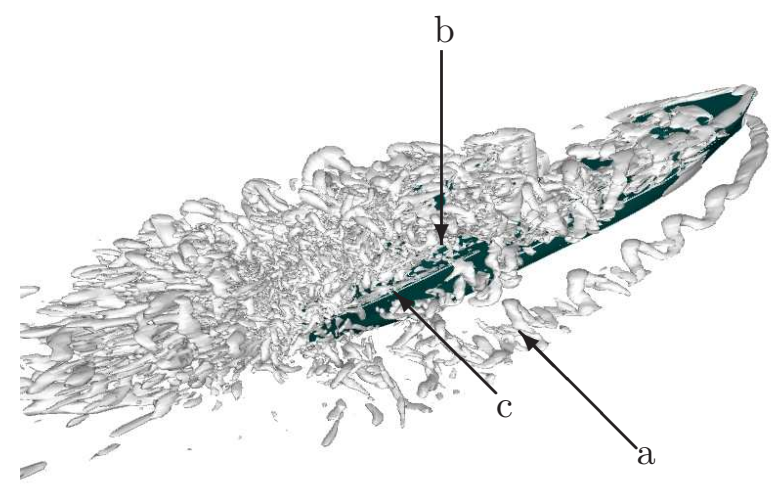

(b) $15^{\circ}$

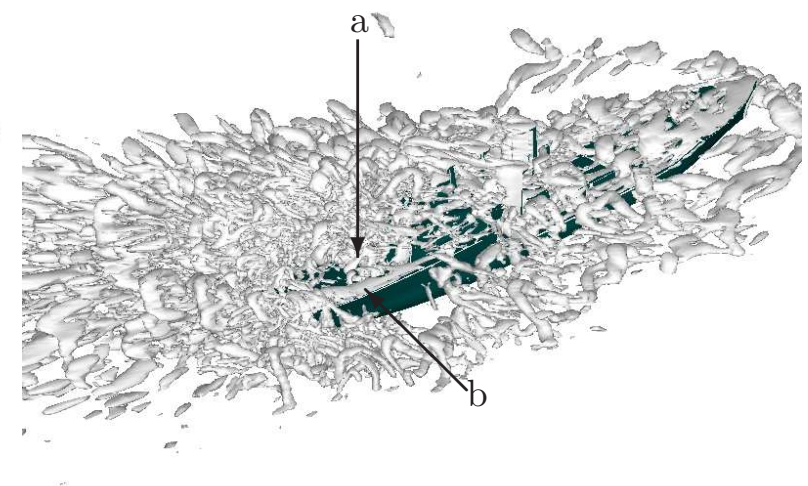

(d) $45^{\circ}$

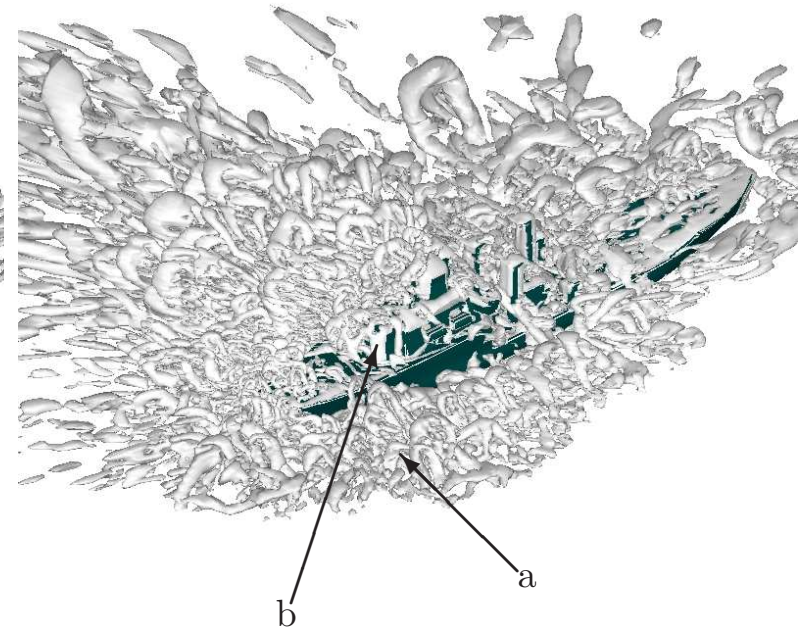

(f) $90^{\circ}$

Figure 11. Visualisations of isosurfaces of $Q$ illustrating the flow features at WOD angles $0^{\circ}$ to $90^{\circ}$ for the Wave Class AO 


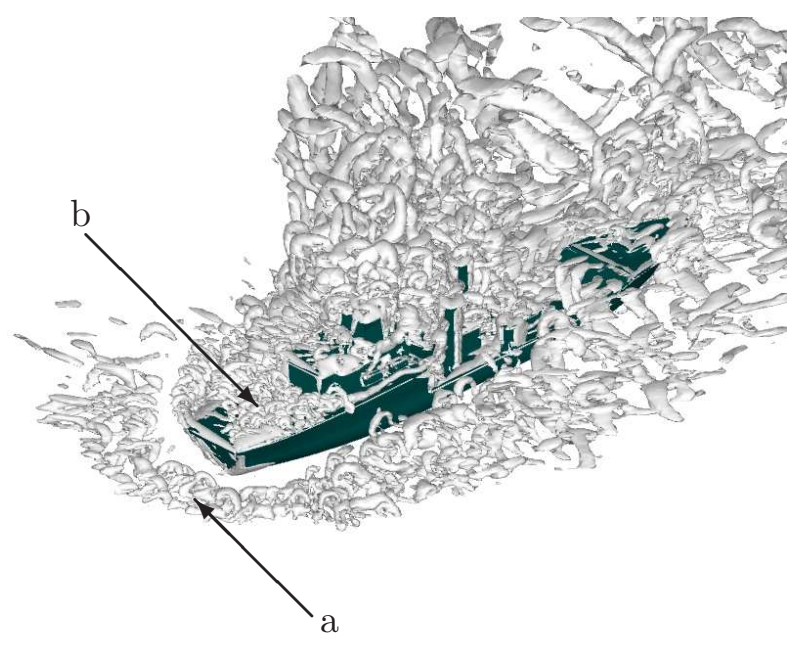

(a) $135^{\circ}$

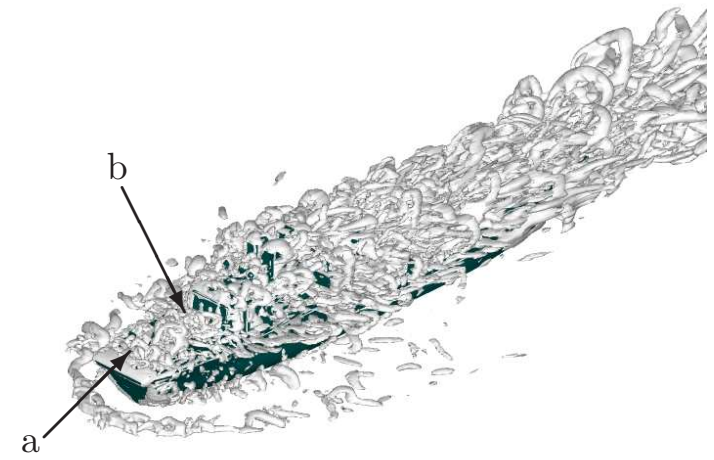

(b) $180^{\circ}$

Figure 12. Visualisations of isosurfaces of $Q$ illustrating the flow features at WOD angles $135^{\circ}$ to $180^{\circ}$ for the Wave Class AO

the mast as described for the $0^{\circ}$ case.

\section{IV.A.3. $30^{\circ}$}

At $30^{\circ}$ the size of the vortices shed from the starboard edge of the ship increases and the long vortex along the starboard side of the ship (below deck level - feature ' $a$ ' in the figure) is larger than at the lower angles of incidence, affecting the flow of air over the top of the ship (acting as a ramp). Large flow structures are ejected periodically from this vortex, passing over the main deck structure. Again, there is a recirculation zone formed behind the superstructure, however now the flow separates on the port side at the bridge and does not reattach along the port side near the location of the lifeboats as it does at lower angles of incidence.

\section{IV.A.4. $45^{\circ}$}

From this angle and higher, flow structure generated by the foredeck appears to have little influence on the airwake in the flight deck and approach region, as it is advected at an angle leading it away from the region of interest. Vortex shedding from the superstructure and lifeboats dominate the flow and there is a large recirculation on the leeward side of the superstructure. The separation from the starboard side and corner of the hangar, and the starboard lifeboat appear to be the principal contributors to turbulence in the flight deck region highlighted as region ' $a$ ' in the illustration. As with the $15^{\circ}$ WOD, there is a strong counter-clockwise rotating vortex beneath the starboard lifeboats which becomes turbulent and expands rapidly, causing strong flow gradients where this meets the turbulent 
flow separated from the back of the hangar (position 'b').

\section{IV.A.5. $60^{\circ}$}

The flow field at this angle is very similar to the flow field at $45^{\circ}$, the principal difference being that the vortex below deck off the starboard side of the ship (between the ship hull and the sea) has split into two vortices, one near the bow of the ship (region 'a') and a second larger vortex starboard of the superstructure (region ' $b$ '). There is a strong recirculation zone to the port side of the flight deck caused by large separation over the superstructure.

\section{IV.A.6. $90^{\circ}$}

This is a rather special WOD angle to consider, as it is exactly perpendicular to the structures on the deck. There is a large recirculation zone between the starboard side of the hull and the sea (feature 'a'), and this aids the flow to pass onto the deck of the ship. Separated flow occurs at the starboard edge of the flight deck, where the energy from the flow passing over the flight deck adds to the longitudinal vortex underneath the lifeboats causing it to increase in size rapidly as it moves aftwards. The separation from the starboard side of the hangar (at point ' $b$ ') results in periodic shedding of vortices at approximately $1.1-1.2 \mathrm{~Hz}$ into the port approach path.

\section{IV.A.7. $135^{\circ}$}

The wind in this case impinges diagonally on the starboard aft corner of the ship, generating a turbulent vortex which wraps around the aft and starboard side of the hull labelled as region 'a'. A shear layer develops over the flight deck characterised by hairpin vortex structures (feature ' $b$ '). These travel diagonally across the landing area, however, the fluctuating velocities are largely restricted to heights of less than $5 \mathrm{~m}$. The shear layer rarely passes higher than this in the region of the landing spot. As with the previous wind angles, the principal contributors to the airwake structure are the hangar, up/intakes, the bridge and radomes - however the small radomes above the bridge appear to have little influence and can most likely be removed from future simulations.

\section{IV.A.8. $180^{\circ}$}

The fully developed flowfield at this angle consists of a shear layer produced at the aft rolling up into hairpin shaped vortices indicated by the label ' $a$ '. There is a horseshoe vortex below deck level behind the ship and some small recirculation at the aft side of the hangar (region ' $b$ ') as the shear layer from the aft passes over the hangar door. Separation occurs at the 
top side of the hangar, and vortex streets are generated from the radomes, up/intake and the mast.

\section{IV.B. Position of the Experimental Measurements}

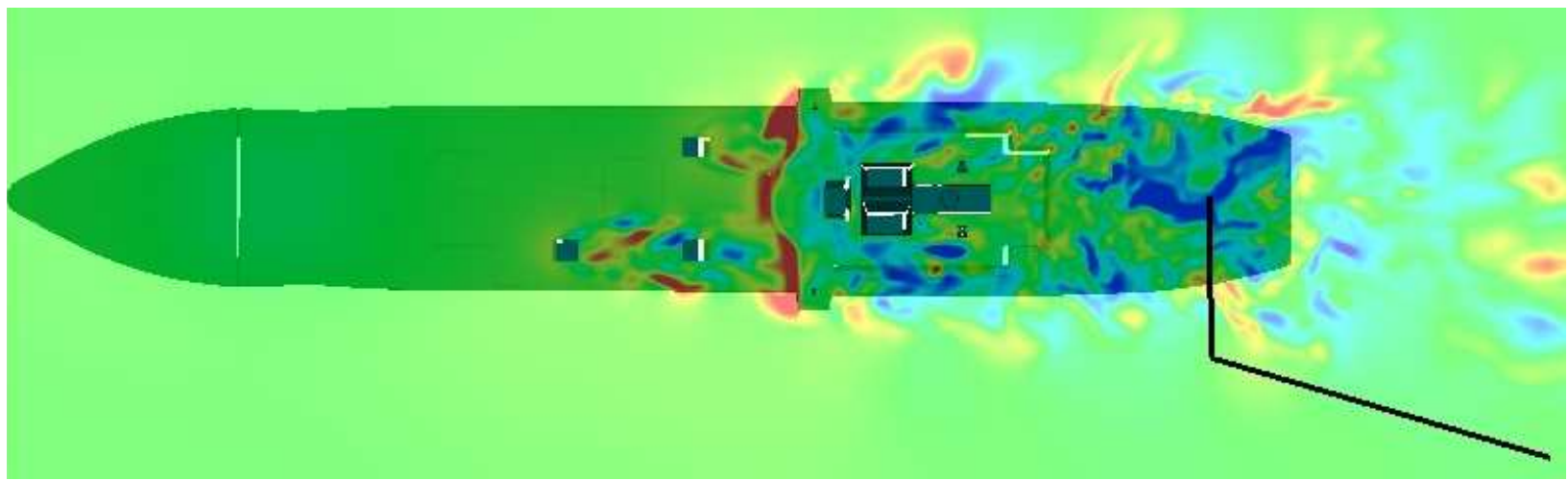

(a) $0^{\circ} \mathrm{WOD}$

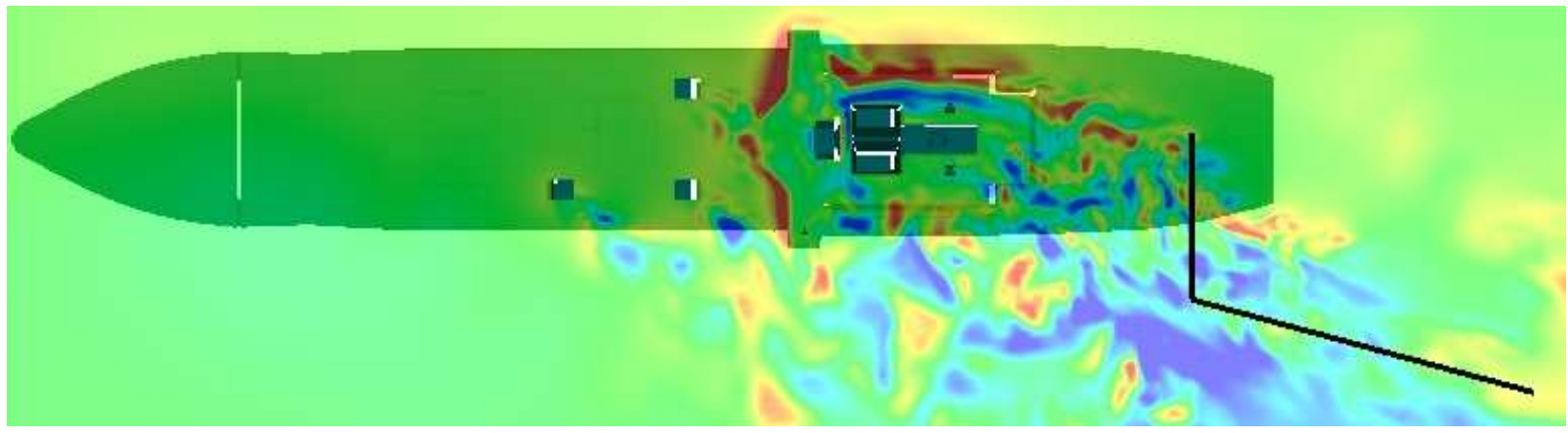

(b) $30^{\circ}$ WOD

Figure 13. Position of the flight path sampled in the LES simulation for comparison with experiment. Contour flood shows the instantaneous vertical velocity component at $z=10 \mathrm{~m}$, illustrating the position of the wake relative to the flight path.

Figure 13 illustrates the line of 25 data points examined in this report, labelled from 1 at the point furthest from the ship to 25 which is above the landing spot.

\section{IV.C. Comparison with Experiment - Ahead $0^{\circ}$}

Figure 14 shows a comparison of the mean flow and fluctuating velocities for 25 points in the approach path for the Wave Class AO with a head wind of 50 knots. As can be seen in Fig. 13 a) the flight path for the $0^{\circ}$ case is on the edge of the wake in the early stages of the approach and then enters the wake for landing.

The mean $u$ and $v$ velocity components are very well estimated, capturing well the small deviations at point 21 and 22 in an excellent quantitative manner. The agreement with the vertical velocities is slightly worse, as the LES predicts a stronger downdraft over the landing deck than is actually present. In this region there are strong fluctuating velocities 


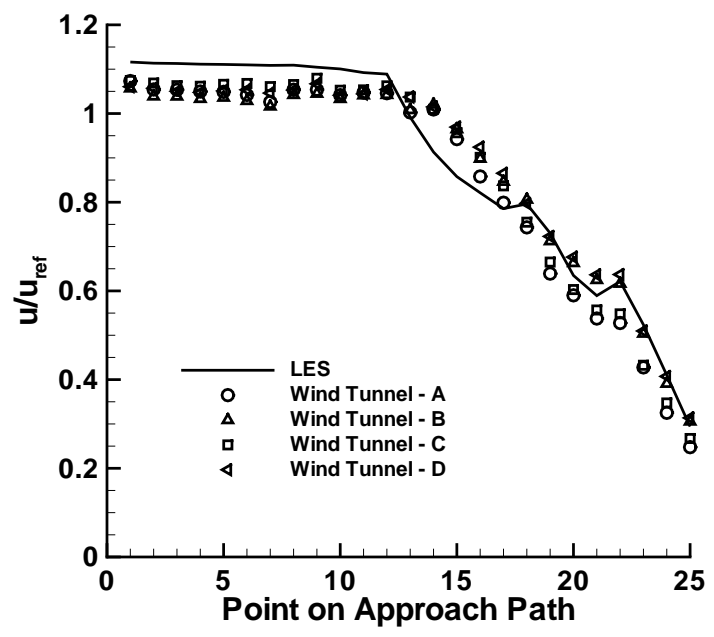

(a) $\mathrm{u}$

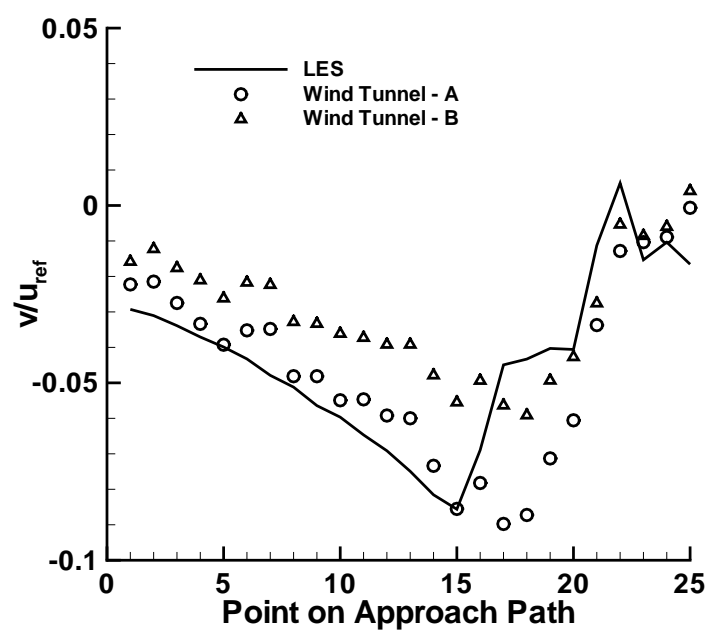

(c) $\mathrm{V}$

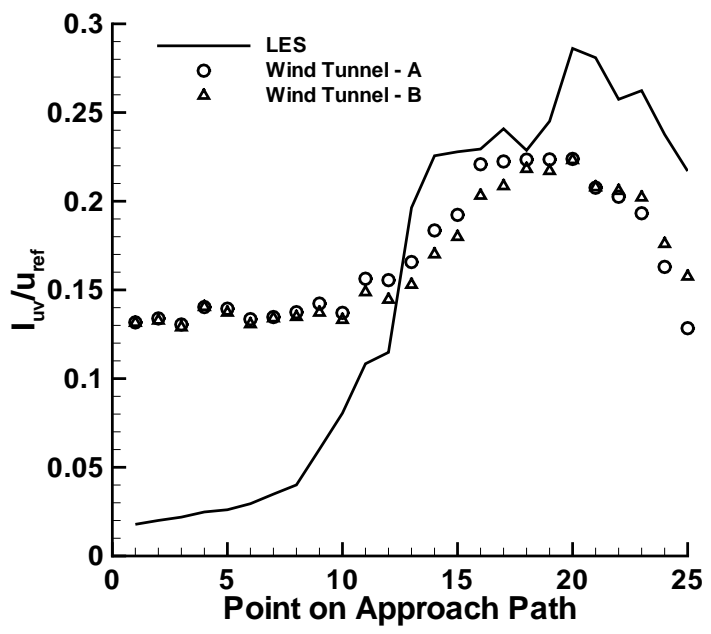

(b) $\sqrt{u^{\prime 2}+v^{\prime 2}}$

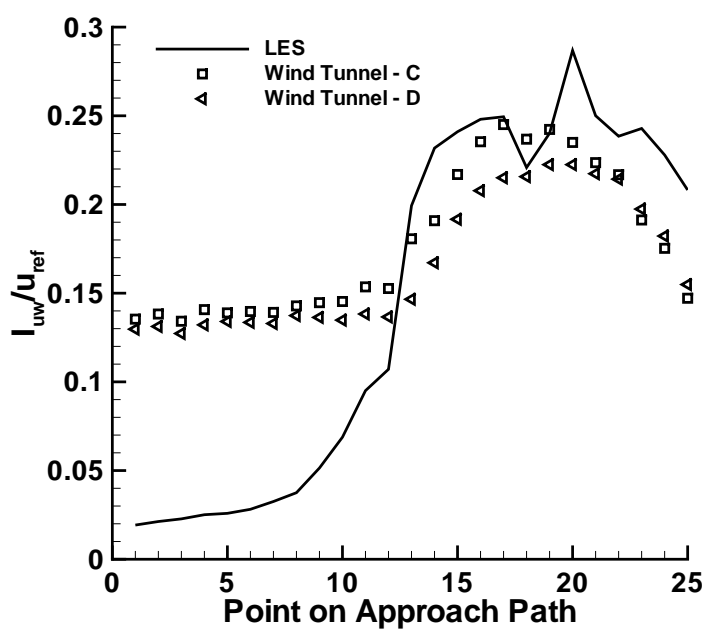

(d) $\sqrt{u^{\prime 2}+w^{\prime 2}}$

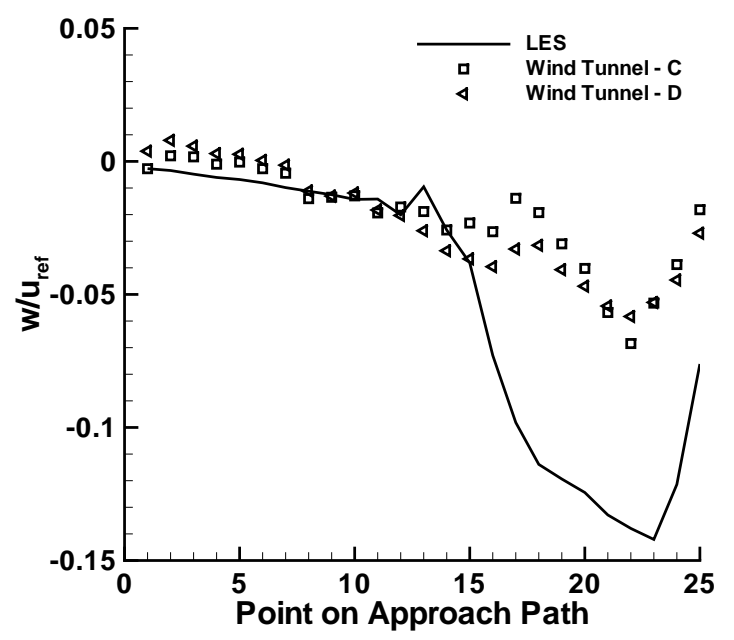

(e) w

Figure 14. Flow properties taken at 25 points along the flight path for the Wave Class AO 
hence the simulation would most likely have to run for a longer physical time for an accurate convergence.

As noted in the first paragraph of this section, the initial stages of the approach until about point 12 are on the edge of the wake. In this region the turbulent fluctuations are dominated by the background turbulent which is not present in the simulation. However, from point $12-25$ when the flight path enters the wake the agreement is excellent, indicating that the production of turbulent close to the ship is well represented.

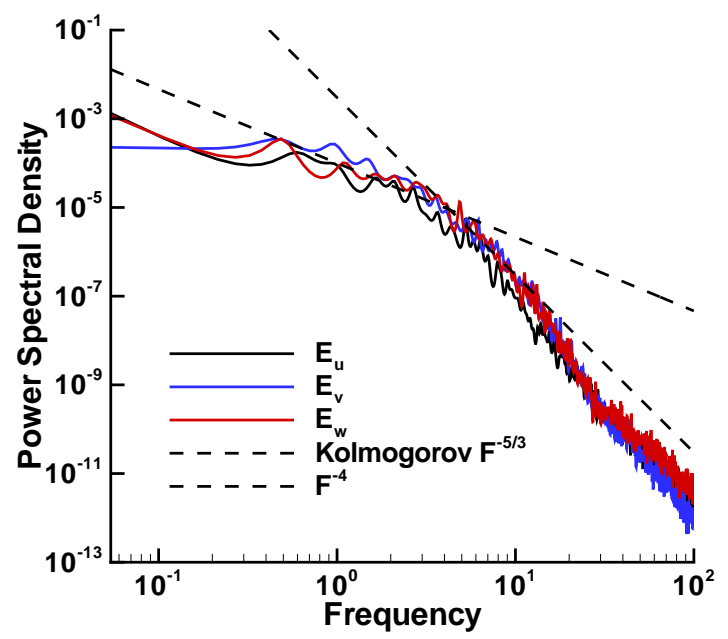

Figure 15. Turbulent power spectra for the $\mathrm{u}, \mathrm{v}$ and $\mathrm{w}$ velocity components at $0^{\circ}$ WOD for the Wave Class AO.

Figure 15 shows the spectra from the three velocity components measured at point 22 on the approach path, coordinates $x=24, y=0, z=10$ (relative to the centre of the hangar door). The spectra have much in common with the Type 23 spectra, including a reasonably large range which varies as $F^{-5 / 3}$, and a kink in the spectra around $4-5 \mathrm{~Hz}$ where it transitions to a $F^{-4}$ power law profile. Interestingly, the power in each of the three components is fairly isotropic, with equal power in each direction as is implied by Figs 14 (b) and (d) where the total magnitude is approximately the same.

\section{IV.D. Comparison with Experiment - Green $30^{\circ}$}

Figure 16 shows a comparison of the mean flow and fluctuating velocities for 25 points in the approach path. As can be seen in Fig. 13 b) the flight path for the $30^{\circ}$ case is in the wake in the early stages of the approach, however as the final manoeuvre is made for landing the helicopter will be located on the edge of the wake in the shear layer from the separation from the starboard side of the hangar.

Considering the mean velocities there is a good agreement in all three components, excepting a region of undershoot in the $u$ velocity profile between points $18-21$. The transition 


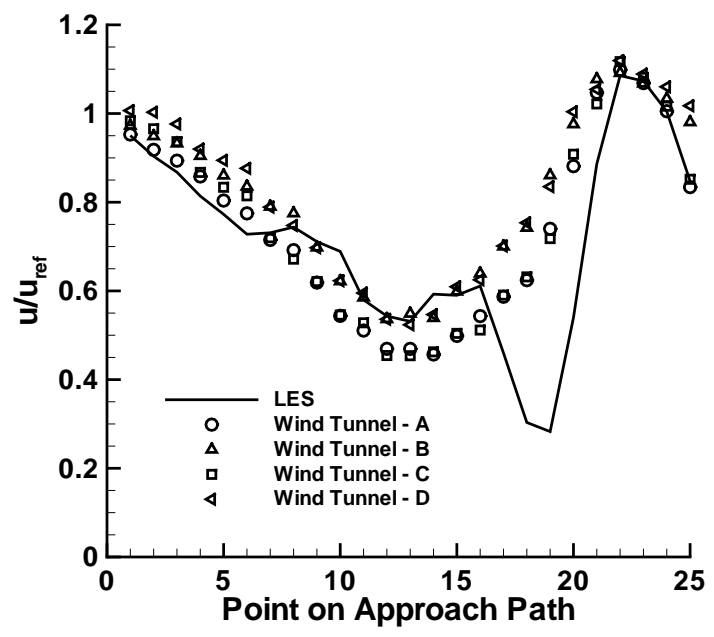

(a) $\mathrm{u}$

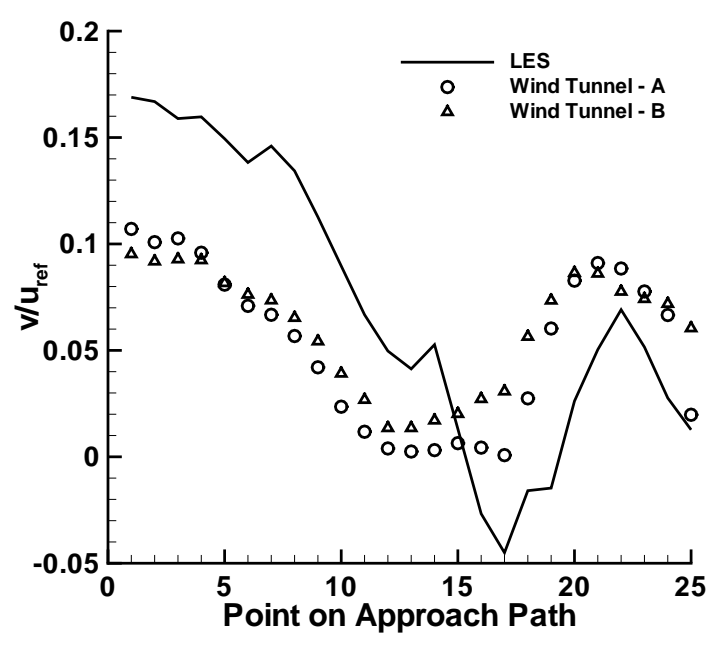

(c) $\mathrm{v}$

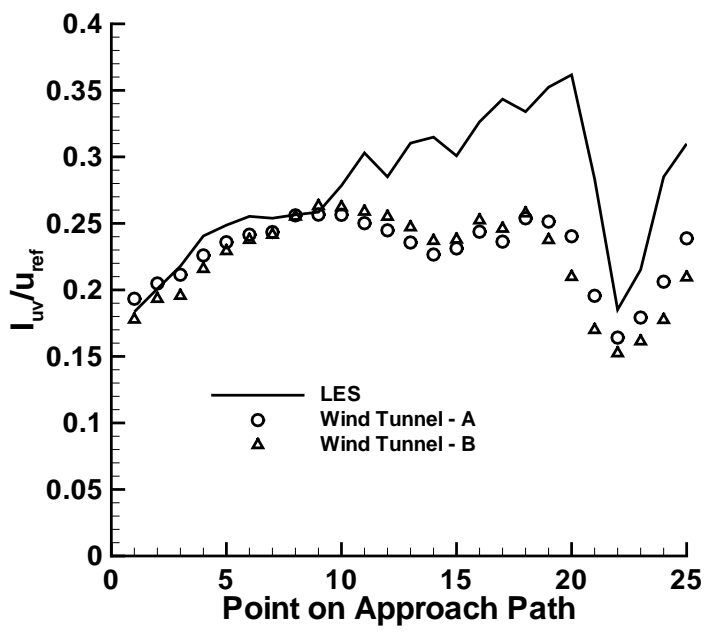

(b) $\sqrt{u^{\prime 2}+v^{\prime 2}}$

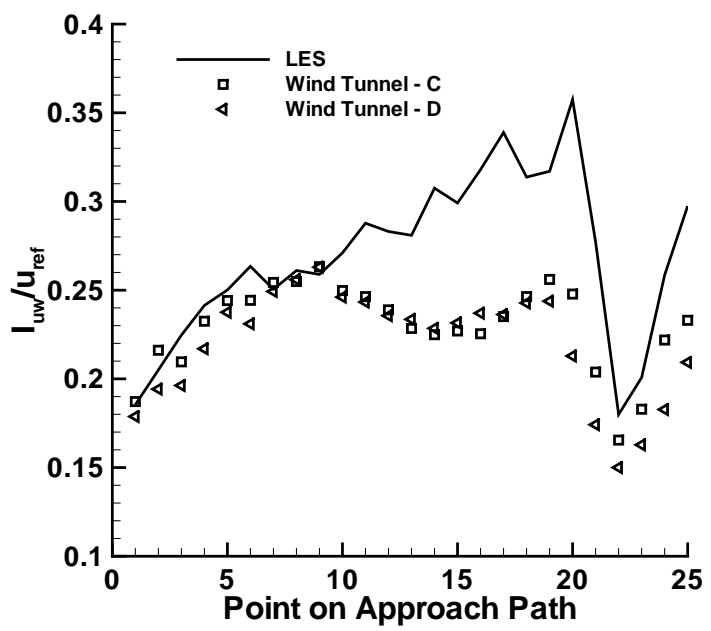

(d) $\sqrt{u^{\prime 2}+w^{\prime 2}}$

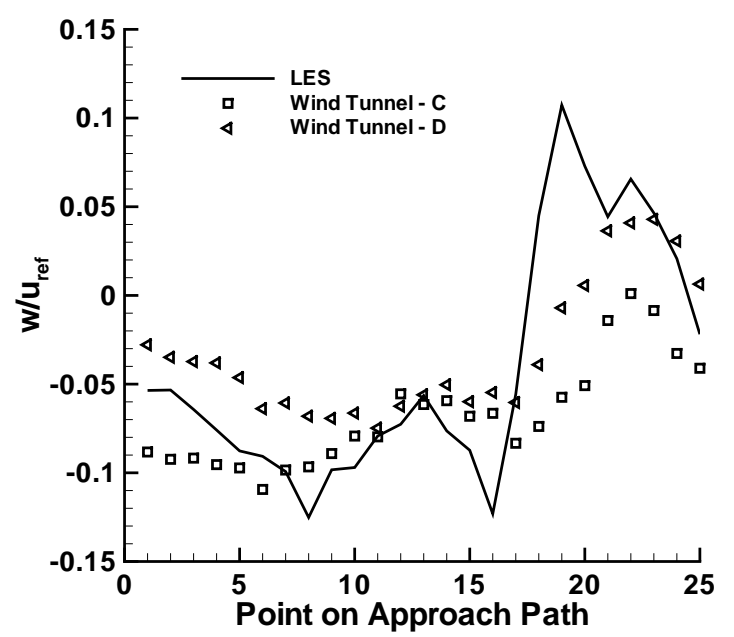

(e) w

Figure 16. Flow properties taken at 25 points along the flight path for the Wave Class AO 
from a downdraft on the port side of the wake to an updraft on the starboard side is predicted well as can be seen in Fig. 16 (e). With regard to the fluctuating components, $I_{u v}$ has the same qualitative shape as the experimental results, however the turbulent kinetic energy predicted is higher than that measured in the experiment. The same observation can be made regarding $I_{u w}$.

The agreement of mean and fluctuating velocities within the airwake suggests that the influence of atmospheric turbulence is not as great as expected from wind-tunnel investigations of simple bluff bodies (such as the results of Saathoff and Melbourne ${ }^{36}$ discussed in Section II.D). Examination of the flow fields (animations of Figs. 11 and 12) shows that there is a significant unsteady flow field caused by the vortex which lies between the side of the ship and the sea surface at most WOD angles. It is conjectured that turbulent fluctuations generated in these vortices generate an effective background turbulence approaching the sharp separation point on the deck of the ship, or on the superstructure. This increased level of turbulent fluctuations is enough to give a reasonably realistic behaviour of the first separation, and hence the development of the airwake.

At WOD angles between $\pm 30^{\circ}$, the turbulent fluctuations over the flight deck are dominated by the wake generated from the forward superstructures, hence once again the turbulence levels are high and thus reasonable recirculation lengths are gained as demonstrated in the location of the transition from downdrafts to updrafts in Fig. 16 (e).

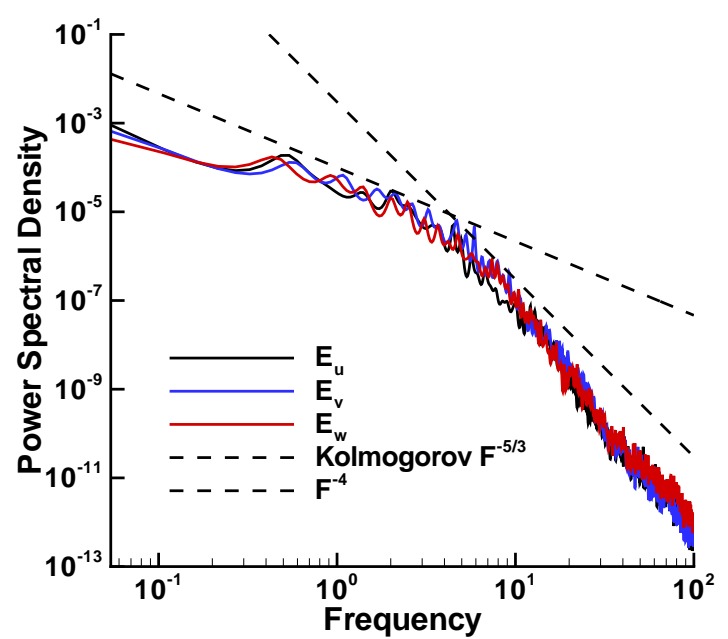

Figure 17. Turbulent power spectra for the u, v and w velocity components at $30^{\circ}$ WOD for the Wave Class AO.

Figure 17 shows the spectra from the three velocity components measured at point 22 on the approach path, coordinates $x=24, y=0, z=10$ (relative to the centre of the hangar door). The shape of the spectrum is very similar to that at $0^{\circ}$, however there is lower total energy in the wake which is to be expected as point 22 in this case is on the edge of the wake 
whereas for the $0^{\circ}$ case it is located in the centre of the wake.

\section{IV.E. WOD Scaling}

Two additional simulations were run at 10 and 30 knots $30^{\circ}$ WOD to check the common assumption that the velocities and their fluctuations are self similar with the WOD velocity, i.e. scaling the flow velocities and the time by $U_{W O D}$ the flow fields are equivalent. This is the same as saying that the flow field is insensitive to variations in Reynolds number $(=\rho u l / \mu)$ in the regime of interest. This wind angle was chosen as historically it is believed to be the most difficult angle to simulation, hence it should be the most sensitive to small differences in Reynolds number.

Figure 18 compares the mean and fluctuating velocities for the three velocity magnitudes investigated. Note that it does not employ the same data points as the experimental investigation. Points 1-11 and 22-25 are the same as the points outputted in the comparison with experiment. Points $12-21$ have the same $x$ and $z$ co-ordinate however the $y$ co-ordinate is reversed - this means that these points are on the starboard side of the boat instead of the port. This was to examine if there was any difference in the laminar regions of the flow field before the airwake.

As can be seen, there is some scatter in the mean values plotted in Fig. 18, although all simulations follow the same trends. What is especially important is that in laminar flow (points $12-21$ ) the results are very close to identical. This implies that the chosen scaling does hold in the range of velocities considered.

In the airwake itself (Points $1-11$ and $22-25$ ) there is some scatter in the data which could be interpreted as meaning that there is a Reynolds number dependence in the simulations. However, Fig. 19a) shows the scaled time varying velocity for the three simulations at point 22 in the airwake - directly over the landing spot. It demonstrates that the simulations are identical for the initial approximately 250 scaled time units (equivalent to $6.25 \mathrm{~s}$ of physical time at 50 knots). This is enough for one full crossing of air over the length of the ship, indicating that there will be a very complex flow present at this time so the fact that the flows remain identical is strong support for WOD scaling. At that point the simulations become different. As turbulence is an incredibly chaotic phenomena then it is not surprising that even a very small change to a source term such as the viscous stresses generates results which differ at late time.

As demonstrated in Fig. 19 b), the power spectral density of the $u$ velocities is practically identical where there are good statistics (i.e. many modes in the given time frame), hence it is concluded that although there are differences between the various WOD velocities, this can be easily due to the the chaotic nature of the turbulent flow itself as the mean flow statistics agree to within the $\pm 10 \%$ as expected from a not-quite converged statistical average. These 


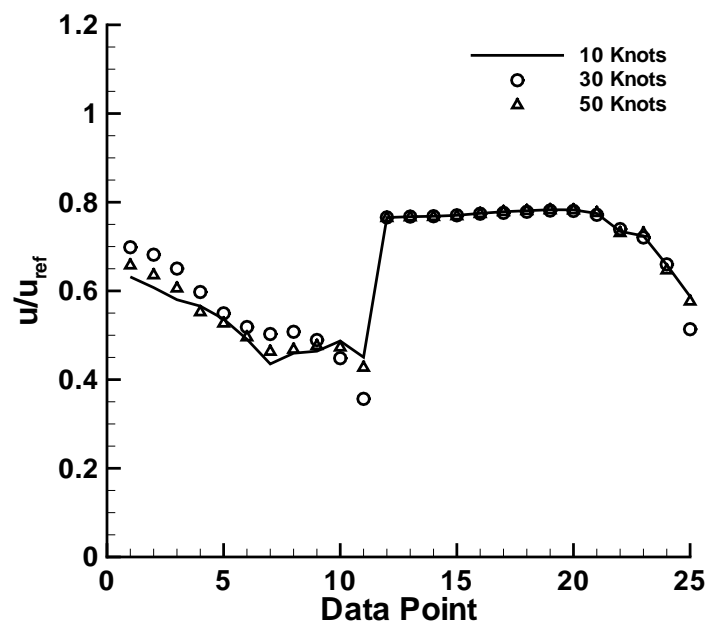

(a) $\mathrm{u}$

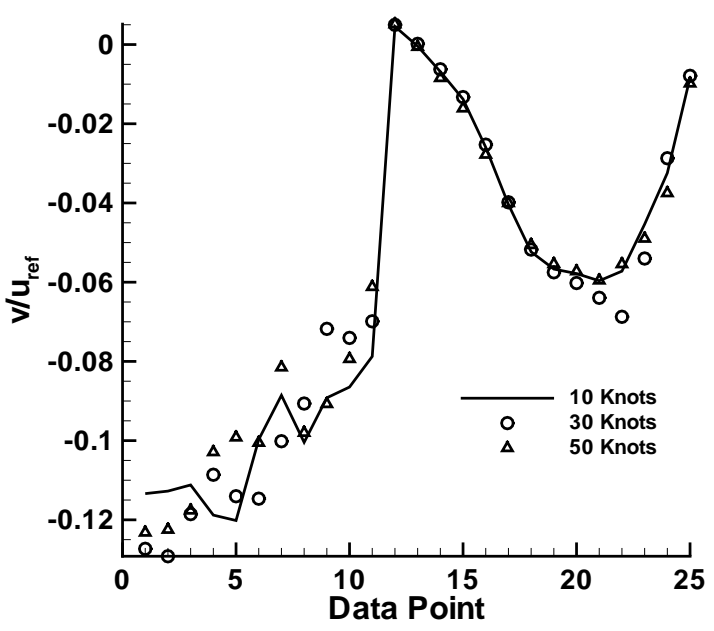

(c) $\mathrm{v}$

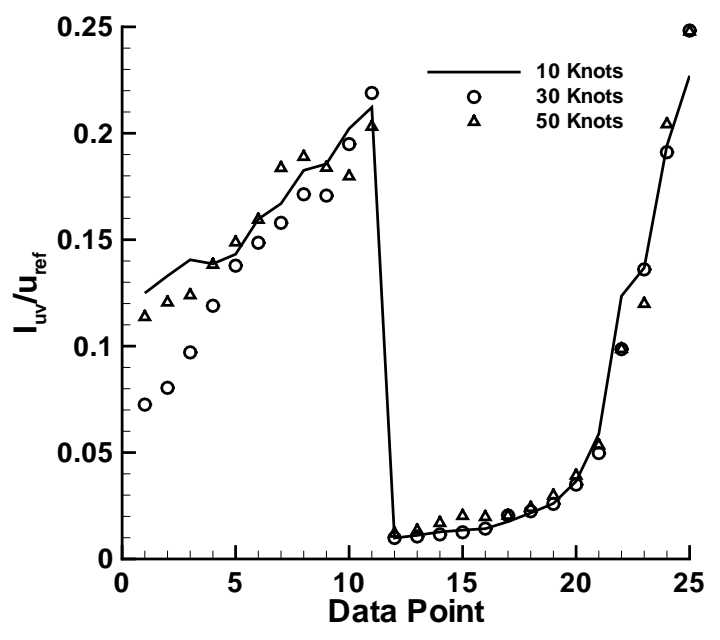

(b) $\sqrt{u^{\prime 2}+v^{\prime 2}}$

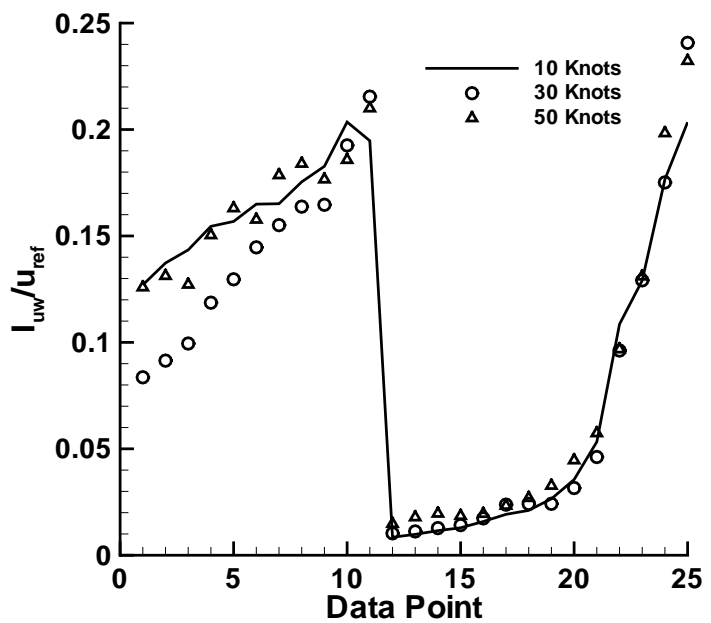

(d) $\sqrt{u^{\prime 2}+w^{\prime 2}}$

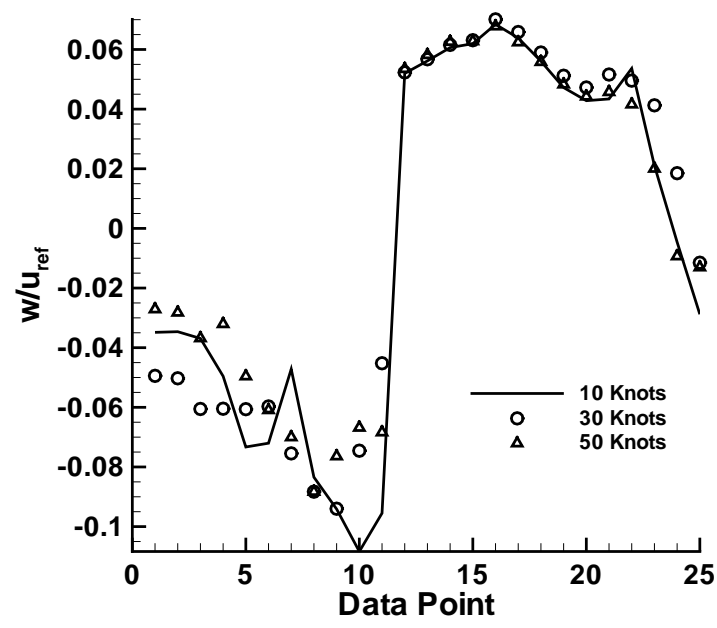

(e) w

Figure 18. Flow properties taken at 25 points to test WOD velocity scaling. Note that points $12-21$ are NOT the same as in the previous plots 


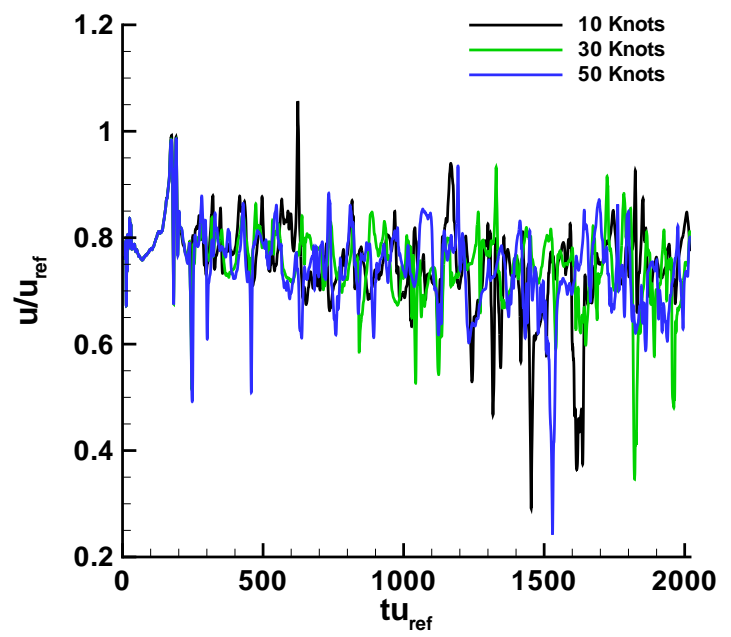

(a) Scaled velocities in scaled time

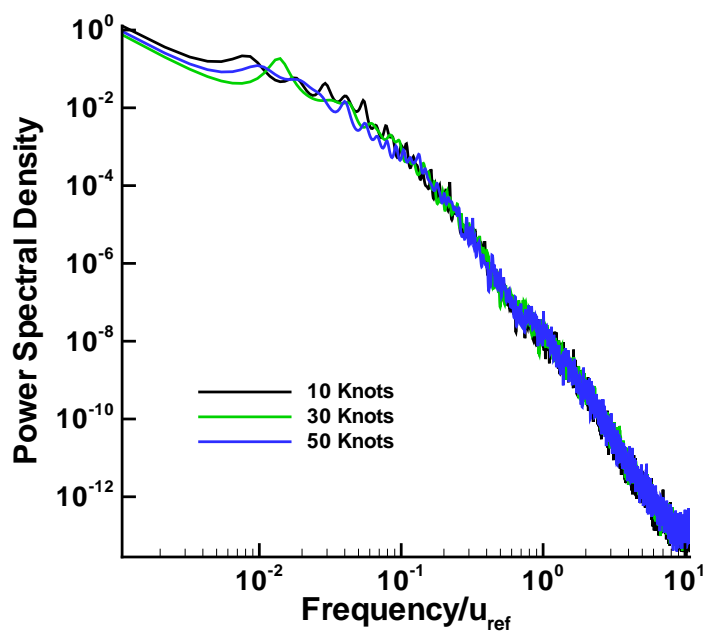

(b) Scaled PSD against scaled frequency

Figure 19. a)Time varying behaviour of the flow at point 22 at three different WOD velocities and their b) PSD of the $u$ velocity component for Point 22

results are in agreement with the observations of Polsky. ${ }^{29}$ 


\section{Conclusions}

This paper has detailed the production of airwake data for two ships and fourteen different wind angles for use in the SAIF project. The qualitative details of the flow field for each ship and wind direction have been discussed and comparisons were made with experiment. Finally, the concept of Wind-Over-Deck scaling was tested.

The airwake over the flight deck is determined by five main flow features. First and most obviously are the large super-structures (e.g. Masts) which generate turbulent wakes. Equally important however are the turbulent vortices which lie between the hull and the sea surface, acting as a 'ramp'. These significantly influence the mean flow over the flight deck and are similar to the horseshoe vortices seen in flow around surface-mounted cubes. ${ }^{28}$ The final three important features are the shear layers which separate from both the sides and top of the hangar, the separation from the edge of the flight deck itself, and the longitudinal vortices along the deck of the ship.

A very good agreement of the mean flow and fluctuating quantities measured in experiments with predictions from the high resolution CFD data is demonstrated for both ships . The greatest discrepancy in the fluctuating components is due to the high level of background turbulence in the wind tunnel experiments which is not represented in the ILES simulation. Once within the wake the ILES prediction is close in magnitude to the experimental measurements. There is also a very good agreement of the mean flow velocities outside the wake indicating that the simulations are accurately representing the blockage effect of the ship.

In addition, excellent agreement with the experimental spectra has been demonstrated, indicating that as well as capturing the magnitude of the fluctuating velocities reasonably well, the distribution of the fluctuating power at different frequencies is also captured. This includes what appears to be a change in the power spectrum at higher frequencies from $P \propto F^{-5 / 3}$ to $P \propto F^{-4}$. This indicates that the mean eddy velocities decrease rapidly above a certain frequency.

An investigation of the WOD scaling at $30^{\circ}$ lead to the conclusion that if there are differences within the range tested, then these differences are masked by the statistical fluctuations due to the chaotic nature of the turbulent wake. Spectral analysis demonstrates that the scaled frequency content of the flows at different WOD are very similar.

Recommendations for future work include an in depth study of flow around simple cube shapes such as reproducing the work of Martinuzzi and Tropea ${ }^{28}$ with the current methods. In this case the work should focus on the upper surface of the cube, to examine the source of the $F^{-4}$ behaviour of the kinetic energy spectrum. Future analysis of realistic ship geometry will include better grid resolution between the hull of the ship and the sea in order to capture the longitudinal vortices more accurately. Ideally the simulation period would also 
be extended from 40s to 400s to find out if this significantly changes the results, and if the very low frequency oscillations recorded in the wind tunnels can be reproduced in the ILES simulations. Finally, it would be important to examine the influence of atmospheric turbulence and surface roughness on the airwake, and the effect of modifications to the ships structure on the airwake properties.

\section{Acknowledgments}

The authors would like to acknowledge the financial support of the MoD. Additionally, we would like to acknowledge technical discussions with John Duncan (MoD), Ian Cox (SEA), Graham Turner (Qinetiq) and Bryan Finlay (DSTL) regarding airwake data generation.

\section{References}

${ }^{1}$ M.C. Bogstad, W.G. Habashi, I. Akel, and D. Ait-Ali-Yahia. Computational-fluid-dynamics based advanced ship-airwake database for helicopter flight simulators. J. Aircraft, 39:830-838, 2002.

${ }^{2}$ J.P. Boris, F.F. Grinstein, E.S. Oran, and R.L. Kolbe. New insights into large eddy simulation. Fluid. Dyn. Res., 10:199-228, 1992. doi:10.1016/0169-5983(92)90023-P.

${ }^{3}$ I.P Castro and A.G. Robins. The flow around a surface-mounted cube in uniform and turbulent streams. J. Fluid. Mech., 77:307-335, 1977. doi:10.1017/S0022112077000172.

${ }^{4}$ R.H. Cohen, W.P. Dannevik, A.M. Dimits, D.E. Eliason, Y. Mirin, A.A.and Zhou, D.H. Porter, and P.R. Woodward. Three-dimensional simulation of a Richtmyer-Meshkov instability with a two-scale initial perturbation. Phys. Fluids, 14(10):3692-3709, 2002.

${ }^{5}$ I. Cox, G. Turner, B. Finlay, and J. Duncan. The ship/air interface framework (SAIF) project: Dynamic challenges. In Maritime Operations of Rotorcraft, 11-12 June 2008.

${ }^{6}$ R.M. Czerwiec and S.A. Polsky. LHA airwake wind tunnel and CFD comparison with and without bow flap. AIAA 2004-4832, 2004.

${ }^{7}$ S.B. Dalziel, P.F. Linden, and D.L. Youngs. Self-similarity and internal structure of turbulence induced by Rayleigh-Taylor instability. J. Fluid Mech., 399:1-48, 1999.

${ }^{8}$ D. Drikakis. Advances in turbulent flow computations using high-resolution methods. Prog. Aerosp. Sci., 39:405-424, 2003. doi:10.1016/S0376-0421(03)00075-7.

${ }^{9}$ D. Drikakis, C. Fureby, F. Grinstein, M. Hahn, and D. Youngs. MILES of transition to turbulence in the Taylor-Green vortex system. In ERCOFTAC Workshop on Direct and Large Eddy Simulation-6, page 133, Sept. 2006.

${ }^{10}$ D. Drikakis, M. Hahn, A. Mosedale, and B. Thornber. Large eddy simulation using high resolution and high order methods. Philos T. R. Soc. A, Accepted, 2009.

${ }^{11}$ D. Drikakis and W. Rider. High-Resolution Methods for Incompressible and Low-Speed Flows. Springer Verlag, Cambridge, 2004.

${ }^{12}$ J.S. Forrest, I. Owen, G.D. Padfield, and S.J. Hodge. Detached-eddy simulation of ship airwakes for piloted helicopter flight simulation. In Proceedings of the International Aerospace CFD Conference, 18-19 June 2007. 
${ }^{13}$ C. Fureby and F.F. Grinstein. Large eddy simulation of high-Reynolds-number free and wall-bounded flows. J. Comput. Phys., 181:68-97, 2002. doi:10.1006/jcph.2002.7119.

${ }^{14}$ C. Fureby, F. Tabor, H.G. Weller, and A.D. Gosman. A comparative study of subgrid scale models in homogeneous isotropic turbulence. Phys. Fluids, 9(5):1416-1429, 1997.

${ }^{15}$ S.K. Godunov. A finite-difference method for the computation of discontinuous solutions of the equations of fluid dynamics. Mat. Sb., 47:271-295, 1959.

${ }^{16}$ R.E. Gordnier and M.R. Visbal. Compact difference scheme applied to simulation of low-sweep delta wing flow. AIAA J., 43(8):1744-1752, 2005.

${ }^{17}$ F.F. Grinstein and C. Fureby. Recent progress on MILES for high Reynolds number flows. J. Fluid Eng.- T. ASME, 848:848-861, 2002. doi:10.1115/1.1516576.

${ }^{18}$ F.F. Grinstein, L.G. Margolin, and W.J. Rider, editors. Implicit Large Eddy Simulation: Computing Turbulent Fluid Dynamics. Cambridge University Press, Cambridge, 2007.

${ }^{19}$ M.J. Guillot and M.A. Walker. Unsteady analysis of the air wake over the lpd-17. AIAA 2000-4125, 2000.

${ }^{20} \mathrm{M}$. Hahn and D. Drikakis. Large eddy simulation of compressible turbulence using high-resolution methods. Int. J. Numer. Meth. Fl., 49:971-977, 2005. doi:10.1002/fld.882.

${ }^{21}$ S. Hickel, N.A. Adams, and J.A. Domaradzki. An adaptive local deconvolution method for implict LES. J. Comput. Phys., 213:413-436, 2006. doi:10.1016/j.jcp.2005.08.017.

${ }^{22}$ J. Jeong and F. Hussain. On the identification of a vortex. J. Fluid. Mech., 285:69-94, 1995.

${ }^{23}$ K.H. Kim and C. Kim. Accurate, efficient and monotonic numerical methods for multi-dimensional compressible flows part II: Multi-dimensional limiting process. J. Comput. Phys., 208:570-615, 2005. doi:10.1016/j.jcp.2005.02.022.

${ }^{24}$ A.N. Kolmogorov. The local structure of turbulence in an incompressible fluid at very high Reynolds numbers. Dokl. Akad. Nauk. SSSR, 30:299, 1941.

${ }^{25}$ M. Lesieur and O. Metais. New trends in large-eddy simulations of turbulence. Annu. Rev. Fluid Mech., 28:45-82, 1996.

${ }^{26}$ L.G. Margolin, P.K. Smolarkiewicz, and Z. Sorbjan. Large-eddy simulations of convective boundary layers using nonoscillatory differencing. Physica D, 133:390-397, 1999. doi:10.1016/S0167-2789(99)00083-4.

${ }^{27}$ L.G. Margolin, P.K. Smolarkiewicz, and A.A. Wyszogrodzki. Implicit turbulence modelling for high Reynolds number flows. J. Fluids Eng., 124:862-867, 2002. doi:10.1063/1.1522379.

${ }^{28}$ R. Martinuzzi and C. Tropea. The flow around surface-mounted, prismatic obstacles placed in a fully developed channel flow. J. Fluids Eng., 115(1):85-92, 1993.

${ }^{29}$ S.A. Polsky. A computational study of unsteady ship airwake. AIAA 2002-1022, 2002.

${ }^{30}$ S.A. Polsky. CFD predication of airwake flowfields for ships experiencing beam winds. AIAA 2003365\%, 2003.

${ }^{31}$ S.B. Pope. Turbulent Flows. Cambridge University Press, 2000.

${ }^{32}$ D.H. Porter, P.R. Woodward, and A. Pouquet. Inertial range structures in decaying compressible turbulent flows. Phys. Fluids, 10(1):237-245, 1998.

${ }^{33}$ Qinetiq. Private Communication, 2007.

${ }^{34}$ K.R. Reddy, R. Toffoletto, and K.R.W. Jones. Numerical simulation of ship airwake. Comput. Fluids, 29:451-465, 2000. 
${ }^{35}$ D.M. Roper, I. Owen, G.D. Padfield, and S.J. Hodge. Integrating CFD and piloted simulation to quantify ship-helicopter operating limits. Aeronaut. J., pages 419-428, July 2006. Paper No. 3051.

${ }^{36}$ P.J. Saathoff and W.H. Melbourne. Effects of free-stream turbulence on surface pressure fluctuations in a separation bubble. J. Fluid Mech., 337:1-24, 1997.

${ }^{37}$ P. Sagaut. Large Eddy Simulation for Incompressible Flows. Springer Verlag, 2001.

${ }^{38}$ N. Sezer-Uzol, A. Sharma, and L.N. Long. Computational fluid dynamics simulations of ship airwake. J. Aerospace Eng, 219(G):369-392, 2005.

${ }^{39}$ A. Sharma and L.N. Long. Airwake simulations on an LPD 17 ship. AIAA 2001-2589, 2001.

${ }^{40}$ J. Shipman, S. Arunajatesan, C. Menchini, and N. Sinha. Ship airwake sensitivities to modeling parameters. AIAA 2005-1105, 2005.

${ }^{41}$ P.K. Smolarkiewicz and L.G. Margolin. MPDATA: a finite difference solver for geophysical flows. J. Comput. Phys., 140(2):459-480, 1998. doi:10.1006/jcph.1998.5901.

${ }^{42}$ R.J. Spiteri and S.J. Ruuth. A class of optimal high-order strong-stability preserving time discretization methods. SIAM J. Num. Anal., 40(2):469-491, 2002. doi =10.1137/S0036142901389025.

${ }^{43}$ G.F. Syms. Numerical simulation of frigate airwakes. Int. J. Numer. Meth. Fluids, 18(2):199-207, 2004. doi: 10.1080/10618560310001634159.

${ }^{44}$ T.C. Tai. Simulation and analysis of LHD ship airwake by Navier Stokes method. In Proceedings of the NATO RTO Symposium on Fluid Dynamics Problems of Vehicles Operating near or in the Air-Sea Interface, 5-8 Oct. 1998.

${ }^{45}$ B. Thornber and D. Drikakis. Implicit large eddy simulation of a deep cavity using high resolution methods. AIAA J., 2008. doi:10.2514/1.36856.

${ }^{46}$ B. Thornber, D. Drikakis, R. Williams, and D. Youngs. On entropy generation and dissipation of kinetic energy in high-resolution shock-capturing schemes. J. Comput. Phys., 227:4853-4872, 2008. doi:10.1016/j.jcp.2008.01.035.

${ }^{47}$ B. Thornber, A. Mosedale, and D. Drikakis. On the Implicit Large Eddy Simulation of homogeneous decaying turbulence. J. Comput. Phys., 226:1902-1929, 2007. doi:10.1016/j.jcp.2007.06.030.

${ }^{48}$ B. Thornber, A. Mosedale, D. Drikakis, D. Youngs, and R. Williams. An improved reconstruction method for compressible flows with low Mach number features. J. Comput. Phys., 227:4873-4894, 2008. doi:10.1016/j.jcp.2008.01.036.

${ }^{49}$ E.F. Toro. Riemann Solvers and Numerical Methods for Fluid Dynamics. Springer-Verlag, Cambridge, 1997.

${ }^{50}$ B. van Leer. Towards the ultimate conservative difference scheme.IV. A new approach to numerical convection. J. Comput. Phys., 23:276-299, 1977. doi:10.1016/0021-9991(77)90094-8.

${ }^{51}$ G. Volpe. Performance of compressible flow codes at low Mach number. AIAA J., 31:49-56, 1993.

${ }^{52}$ D.L. Youngs. Three-dimensional numerical simulation of turbulent mixing by Rayleigh-Taylor instability. Phys. Fluids A, 3(5):1312-1320, 1991.

${ }^{53}$ D.L. Youngs. Application of MILES to Rayleigh-Taylor and Richtmyer-Meshkov mixing. AIAA-20034102, June 2003.

${ }^{54}$ D.L. Youngs and R.J.R. Williams. Turbulent mixing in spherical implosions. accepted, Int. J. Numer. Meth. Fl., 2007.

${ }^{55}$ S. Zan. Technical comment on 'computational-fluid-dynamics based advanced ship-airwake database for helicopter flight simulators'. J. Aircraft, 40(5):1007, 2003. 
${ }^{56}$ F. Zhang, H. Xu, and N.G. Ball. Numerical simulation of unsteady flow over SFS 2 ship model. AIAA 2009-81, 2009. 